\title{
Stability and transition between vortex configurations in square mesoscopic samples with antidots
}

\author{
G. R. Berdiyorov, B. J. Baelus, M. V. Milošević, and F. M. Peeters* \\ Departement Natuurkunde, Universiteit Antwerpen (Campus Drie Eiken), Universiteitsplein 1, B-2610 Antwerpen, Belgium
}

(Received 3 July 2003; published 17 November 2003)

\begin{abstract}
Using the complete nonlinear Ginzburg-Landau theory, we investigated the superconducting state and phase boundaries for mesoscopic square samples containing one to four submicron antidots in the presence of a uniform perpendicular magnetic field. The properties of the different vortex states, possible degeneracies, and the transitions between them are studied. Due to the interplay of the different types of symmetry, a qualitative difference in the nucleation of the superconducting state in samples with different number or arrangement of antidots is found. The superconducting/normal state $H-T$ phase boundary of these structures reveals an oscillatory behavior caused by the formation of different stable vortex configurations in these small clusters of pinning centers (antidots). We analyze the stability of these configurations and compare the superconducting phase boundary with experimental results.
\end{abstract}

DOI: 10.1103/PhysRevB.68.174521

PACS number(s): 74.20.De, 74.25.Dw, 74.25.Ha

\section{INTRODUCTION}

Recent progress in microfabrication and measurement techniques made it possible to study the properties of superconducting samples with sizes comparable to the penetration depth $\lambda$ and the coherence length $\xi$. In this case the properties of a superconductor are considerably influenced by confinement effects. Therefore, for such mesoscopic samples nucleation of the superconducting state depends strongly on the boundary conditions imposed by the sample shape, ${ }^{1}$ i.e., on the topology of the system.

Recent technical and theoretical advances have led to a revival of the interest in the magnetic properties of superconducting networks and artificially structured superconducting films. Many different topologies have been studied experimentally and theoretically, which can be classified into simple single loops, ${ }^{1-6}$ multiloop structures, ${ }^{7-15}$ and large infinite networks. ${ }^{16-18}$ Those structures are considered, e.g., for single flux quantum logic applications in analogy with semiconducting $2 \times 2$ quantum dot systems. ${ }^{19}$ In a superconducting $(2 \times 2)$-antidot cluster, the vortices play the role of the electrons in the quantum dots and the antidots play the role of the quantum dots. The advantage of such a system is that the different vortex states can be studied on a macroscopic level and they can even be visualized. ${ }^{20,21}$ Also, these superconducting structures have attracted attention as potential new components for low-temperature electronics.

The theories used to explain experimental results for the $H(T)$ boundary of these structures were mainly based on the linearized Ginzburg-Landau theory, using either the London limit, ${ }^{22,23}$ where the modulus of the order parameter is assumed to be spatially constant, or the de Gennes-Alexander formalism, ${ }^{7,24}$ allowing $|\Psi(x)|$ to vary along (but not across) the strands.

Fomin et al. ${ }^{5}$ studied the superconducting state in a narrow mesoscopic square loop and analyzed phase boundaries on the basis of a self-consistent solution of the GinzburgLandau equations. Baelus and Peeters ${ }^{3}$ considered mesoscopic superconducting disk structures containing a circular antidot and investigated the vortex structures and the $\mathrm{H}-\mathrm{T}$ phase boundary using the complete Ginzburg-Landau formalism.

For the superconducting structures, containing a large number of submicron holes (antidots), it is not a simple exercise to take into account the vortex-vortex interaction due to the very large number of interacting vortices. From this point of view, a microdot with an antidot cluster $(2 \times 2,3$ $\times 3$, etc.) with a small number of interacting vortices is a very promising "intermediate" system between a single superconducting loop with a finite strip width ${ }^{3}$ and a superconducting film with a large regular array of antidots. The reduced number of interacting vortices simplifies the calculations and the results may be extrapolated to the analysis of the vortex state in substantially larger antidot arrays.

In the last decade, several experimental studies ${ }^{11-13}$ were published, where the vortex state of superconducting (2 $\times 2$ )-antidot clusters made of different kinds of superconducting material were investigated. In Ref. 12 the authors studied the transport properties of a superconducting $\mathrm{Pb} / \mathrm{Cu}$ microdot with a $(2 \times 2)$-antidot cluster, measuring the $S / N$ phase boundary, the magnetoresistance, the critical currents, and the $V(I)$ characteristics. They compared their experimental results with calculations in the London limit of the Ginzburg-Landau (GL) theory and in the framework of the de Gennes-Alexander model. It was shown that vortices can be pinned by the antidots forming a cluster and that the ground-state configurations of the vortices are noticeably modified by the current sent through the structure. The authors of Ref. 13 considered a $2 \times 2$ aluminum antidot cluster and a microsquare containing two submicron holes. It was found that the $S / N$ phase boundary $T_{c}(H)$ of these structures shows quite different behavior in low and high magnetic fields.

In this work, we investigated systematically the superconducting state of mesoscopic square samples in the presence of a uniform perpendicular magnetic field for six different topologies (Fig. 1). It is well known that different geometries of mesoscopic superconductors will favor different arrangements of vortices and will make certain vortex configurations more stable than others. Here, we investigate how the vortex configuration and the critical parameters are influenced by 

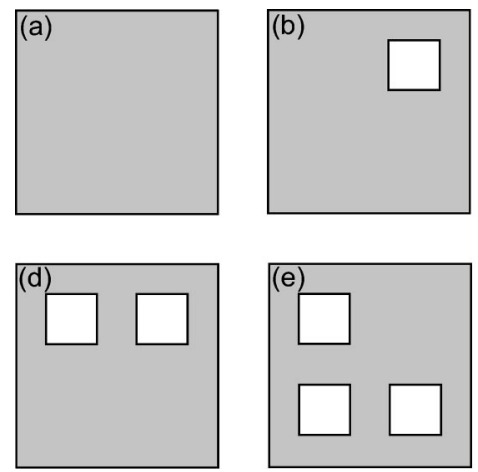

FIG. 1. Model configurations: full microsquare (the reference sample) (a), superconducting squares with one (b), two (c,d), three (e), and (f) four antidots. $W$ denotes the size of the side of the samples, $W_{i}$ is the side of holes, and $W_{0}$ is the distance between antidots. $\kappa$ is the Ginzburg-Landau parameter, and the sample thickness equals $d=0.1 \xi$.

the sample geometry. We studied (see Fig. 1) a filled microsquare (a), a square with one (b), two (c,d), three (e) and four (f) identical antidots, where each antidot can in principle contain a different number of flux quanta.

Our theoretical analysis is based on a full self-consistent numerical solution of the coupled nonlinear GL equations. As an example we took the Ginzburg-Landau parameter $\kappa$ $=0.28$, which is typical for Al thin disks. ${ }^{25}$ No a priori shape or arrangement of the vortex configuration is assumed. The magnetic-field profile near and in the superconductor is obtained self-consistently, and therefore the full demagnetization effect is included in our approach. We calculated quantities such as the free energy, the magnetization, the Cooperpair density, the total magnetic-field profile, and the currentdensity distribution. Due to the interplay of the different kinds of symmetry, there exists a qualitative difference in the nucleation of the superconducting state in samples with different number of holes (antidots).

We calculated the $H-T$ superconducting phase boundary, which shows characteristic oscillations at specific values of the magnetic flux coming from the limited number of possible vortex configurations. A comparison between the calculated $H-T$ phase diagram and the experimental data ${ }^{13}$ confirms that these effects are due to fluxoid quantization and vortex pinning at the antidots. Fluxoid quantization around each antidot leads to characteristic minima in $T_{c}(H)$, corresponding to the transitions between different vortex states, known as Little-Parks oscillations. ${ }^{26}$

In small systems vortices may overlap so strongly that it is more favorable to form one big giant vortex. In order to see clear multivortex configurations we chose sufficiently large values for the sizes of the samples. A square sample with side $W=7.0 \xi$ is taken as a reference sample [Fig. 1(a)]. The dimensions of the holes for all structures were taken the same $W_{i}=2.0 \xi$, and $W_{0}=1.0 \xi$ is the lateral distance between the antidots. The thickness of all samples is taken $d$ $=0.1 \xi$.

The paper is organized as follows. The theoretical formulation of the problem is presented in Sec. II. Different vortex configurations and their stability are studied in Secs. III and
IV. Transitions between these vortex states are presented in Sec. V. In Sec. VI we show the dependence of the free energy and magnetization on the sample parameters, such as the Ginzburg-Landau parameter and the sample thickness. In Sec. VII we present the superconducting/normal phase boundary for the $(2 \times 2)$ antidot cluster. The $H-T$ diagram of this structure is compared with experimental results. Our results are summarized in Sec. VIII.

\section{THEORETICAL FORMALISM}

In the present paper, we consider thin flat superconducting samples of different geometry which are immersed in an insulating medium in the presence of a perpendicular uniform magnetic field $H_{0}$. To solve this problem we follow the numerical approach of Schweigert and Peeters. ${ }^{27,28}$ For thin superconducting samples $(d<\xi, \lambda)$ it is allowed to average the GL equations over the sample thickness for superconductors of arbitrary geometry. Using dimensionless variables and the London gauge $\operatorname{div} \vec{A}=0$ for the vector potential $\vec{A}$, we write the system of GL equations ${ }^{29-31}$ in the following form

$$
\begin{gathered}
\left(-i \vec{\nabla}_{2 \mathrm{D}}-\vec{A}\right)^{2} \Psi=\Psi\left(1-|\Psi|^{2}\right), \\
-\Delta_{3 D} \vec{A}=\frac{d}{\kappa^{2}} \delta(z) \vec{j}_{2 \mathrm{D}},
\end{gathered}
$$

where

$$
\vec{j}_{2 \mathrm{D}}=\frac{1}{2 i}\left(\Psi^{*} \vec{\nabla}_{2 \mathrm{D}} \Psi-\Psi \vec{\nabla}_{2 \mathrm{D}} \Psi^{*}\right)-|\Psi|^{2} \vec{A},
$$

is the density of the superconducting current. The indices 2D and $3 \mathrm{D}$ refer to two- and three-dimensional operators, respectively. The superconducting wave function satisfies the boundary conditions $\left.\left(-i \vec{\nabla}_{2 D}-\vec{A}\right) \Psi\right|_{n}=0$ at the sample surface and the vector potential is given by $\vec{A}=\frac{1}{2} H_{0} \rho \vec{e}_{\phi}$ far away from the superconductor. Here the distance is measured in units of the coherence length $\xi$, the vector potential in $c \hbar / 2 e \xi$, and the magnetic field in $H_{c 2}=c \hbar / 2 e \xi^{2}=\kappa \sqrt{2} H_{c}$. The thin flat superconductor is placed in the $(x, y)$ plane, and the external magnetic field is directed along the $z$ axis.

To solve the system of Eqs. 1(a,b), we generalized the approach of Ref. 28 for circular disks to flat superconductors with an arbitrary geometry. We apply a finite-difference representation for the order parameter and the vector potential on a uniform $(x, y)$ Cartesian space grid, with typically 128 $\times 128$ grid points for the area of the superconductor, and use the link variable approach ${ }^{33}$ and an iteration procedure based on the Gauss-Seidel technique to find $\Psi$. The vector potential is obtained with the fast Fourier-transform technique where we set $\vec{A}_{|x|=R_{S},|y|=R_{S}}=H_{0}(x,-y) / 2$ at the boundary of the square simulation region with width typically four times the width of the superconductor.

Contrary to circular configurations such as disks ${ }^{28}$ for nonaxially symmetric systems there exist no axially symmetric giant vortex states and hence the superconducting state is always a mixture of different angular harmonics. The vortic- 
ity $L$ of a particular superconducting sample can be calculated by considering the phase $\varphi$ of the order parameter along a closed loop at the boundary of the sample, where the total phase difference is always $\Delta \varphi=2 \pi L$. In nonaxially symmetric systems three possible vortex states exist: (i) a multivortex state that contains separate vortices, (ii) a superconducting state that contains one giant vortex in the center, and (iii) a state that is a mixture of both (a giant vortex in the center which is surrounded by single vortices). The giant vortex is not necessarily circular symmetric as in the case of a circular disk, but it may be deformed due to the specific shape of the sample boundary.

To find the different vortex configurations, which include the metastable states, we search for the steady-state solutions of Eqs. 1 $(a, b)$ starting from different randomly generated initial conditions. Then we increase/decrease slowly the magnetic field and recalculate each time the exact vortex structure. We do this for each vortex configuration in a magneticfield range where the number of vortices stays the same. By comparing the dimensionless Gibbs free energies of the different vortex configurations

$$
F=V^{-1} \int_{V}\left[2\left(\vec{A}-\vec{A}_{0}\right) \cdot \vec{j}_{2 d}-|\Psi|^{4}\right] d \vec{r},
$$

where integration is performed over the sample volume $V$, and $\vec{A}_{0}$ is the vector potential of the uniform magnetic field, we find the ground state, the metastable states, and the magnetic-field range over which the different states are stable. The free energy will be expressed in units of $H_{c}^{2} V / 8 \pi$. The dimensionless magnetization, which is a direct measure of the expelled magnetic field from the sample, is defined as

$$
M=\frac{\langle H\rangle-H_{0}}{4 \pi},
$$

where $H_{0}$ is the applied magnetic field. $\langle H\rangle$ is the magnetic field averaged over the superconducting area of the sample, and $\vec{H}=\operatorname{rot} \vec{A}$. We also calculated the magnetization averaging the field over the $W \times W$ area (superconductor and holes).

The temperature is indirectly included in the calculation, through $\xi, \lambda, H_{c 2}$, whose temperature dependence is given by

$$
\begin{gathered}
\xi(T)=\frac{\xi(0)}{\sqrt{\left|1-T / T_{c 0}\right|}}, \\
\lambda(T)=\frac{\lambda(0)}{\sqrt{\left|1-T / T_{c 0}\right|}}, \\
H_{c 2}(T)=H_{c 2}(0) \mid 1-\frac{T}{T_{c 0} \mid},
\end{gathered}
$$

where $T_{c 0}$ is the critical temperature at zero magnetic field. We will only explicitly insert the temperature dependence if we consider the $H-T$ phase diagrams, while the other calcu- lations are done for a fixed temperature. Notice that the Ginzburg-Landau parameter $\kappa=\lambda / \xi$ is temperature independent.

\section{FREE ENERGY, MAGNETIZATION AND STABILITY OF THE DIFFERENT VORTEX STATES}

The free energy and magnetization of the mesoscopic superconducting samples give us plenty of information on the physical processes in the superconductor, and therefore we will first compare the free energy and magnetization for our different superconducting samples.

Figures 2(a)-2(f) show the free energy for the reference sample (full square) and for the square superconductor with one, two, three, and four holes, respectively as a function of the applied magnetic field. The insets in all figures show an enlargement of the free energy of the states with large vorticity. Open circles indicate continuous transitions and open squares indicate the $S / N$ transition fields. In the reference sample [Fig. 2(a)] vortex states up to $L=11$ can nucleate. At lower magnetic-fields states with vorticity $L=2,3,4,5$, and 6 are multivortex states (see Ref. 32). Further increase of the magnetic field leads to the formation of the giant vortex state in the center of the sample.

The insertion of one hole in the sample changes the free energy of the sample considerably [Fig. 2(b)]. In this case all ground-state transitions between different vortex states occur at lower magnetic fields. The $L=1$ state is stable over a much larger magnetic-field range than the other states. Notice also that the superconducting/normal transition field is larger. This field is $H_{0} / H_{c 2}=2.01$ for the reference sample and $H_{0} / H_{c 2}=3.21$ for the superconductor with a hole. The $60 \%$ increase of the critical magnetic field is completely due to the narrow superconducting area in the upper right corner of the sample. It is well known that superconductivity is enhanced $^{34}$ in such corners. In both cases, only transitions between successive $L$ states are present (i.e., $L=n \rightarrow L=n$ +1 transitions). The maximum vorticity that can be accommodated in the full square is $L=11$, while for the sample with a single hole, this is 19 . The transitions between vortex states after the $L=10$ state are continuous and the position of the transitions at which the vorticity $L$ changes by one unit are indicated by the open dots. The continuous vortex transitions are a consequence of the noncircular geometry of the sample and are analogous to those found earlier in ringlike structures with nonuniform width. ${ }^{3,4}$

Figure 2(c) shows the free energy of the superconducting sample with two antidots, where the antidots are located along the diagonal. In this case there exist vortex states with vorticity up to $L=18$ with a $S / N$ transition field of $H_{0} / H_{c 2}=3.22$ which is almost identical to that of the singleantidot sample. Contrary to the previous two cases, here we found with increasing magnetic field $\Delta L=2$ transitions such as $L=2 \rightarrow L=4, L=6 \rightarrow L=8, L=12 \rightarrow L=14, L=14 \rightarrow L$ $=16$, and $L=16 \rightarrow L=18$. Notice that the last three transitions are continuous and for these transitions $\Delta L=2$; i.e., states with vorticity $L=13,15,17$ do not become the ground state. The energies of all superconducting states are lower and the ground-state transitions occur at lower magnetic 


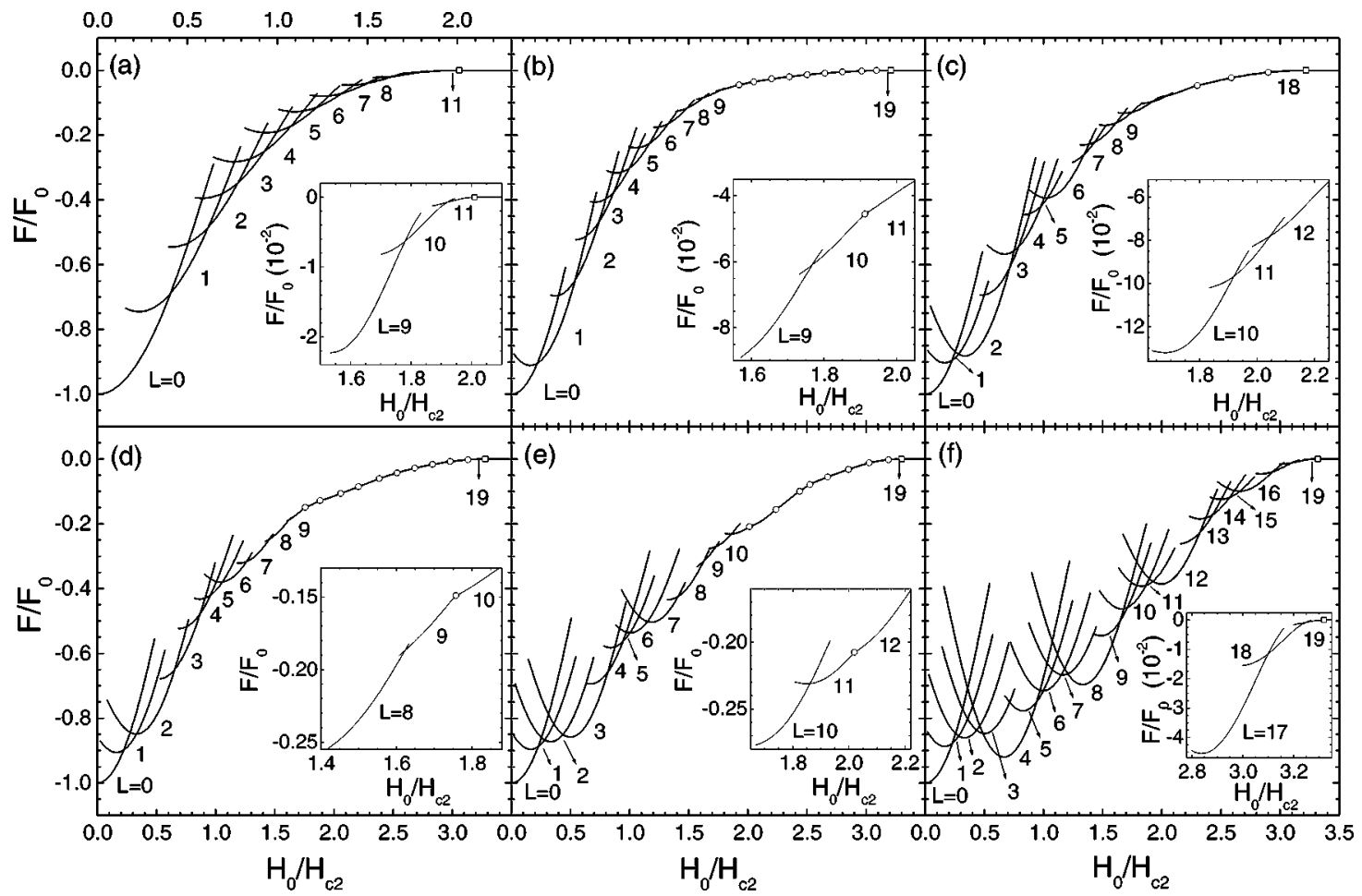

FIG. 2. The free energy as a function of the applied magnetic field for the reference sample (a) and for the square with one (b), two diagonal (c), two top (d), three (e), and four (f) holes. The insets show the free energy for higher vorticity. The open circles indicate continuous transitions between different vortex states and open squares show the $S / N$ state transition fields.

fields as compared to the previous sample. Notice also that the superconducting state with even vorticity is more stable than those with odd vorticity.

In order to see the influence of the sample topology, we considered the superconductor with two antidots, when they are in the top half part of the sample [see Fig. 1(d)]. Figure 2(d) shows the free energy of this structure. In this case the free energy of the states with $2 \leqslant L \leqslant 6$ is higher than the free energy of the sample with the two antidots located along the diagonal. We also found that the transitions after the $L=9$ state are continuous. The maximal number of vortices is $L$ $=19$ and the $S / N$ transition field is $H_{0} / H_{c 2}=3.28$ slightly higher than in previous sample. Notice, that here $\Delta L=1$ between the different vortex states which is a consequence of the different symmetry of the sample as compared to that of Fig. 1(c).

The free energy for the three-antidot sample is shown in Fig. 2(e). In this case we found only $L=n \rightarrow L=n+1$ transitions, except $L=0 \rightarrow L=2$ transition, and in the high magnetic-field region continuous transitions are found after the $L=11$ state.

The most interesting case is the superconductor with four antidots. The free energy of this sample is given in Fig. 2(f). Notice that each of the vortex states has a larger stability region, the energies of the different superconducting states are lower as compared to all previous cases, the transitions between different $L$ states occur at lower magnetic fields; and all thermodynamic equilibrium transitions are discontinuous with $\Delta L=1$. Vortex states with vorticity up to $L=19$ can be nucleated and the $S / N$ transition field $H_{0} / H_{c 2}=3.32$ is the highest of all the six considered structures. There is also a clear enhancement of the stability for states with $L=4,8$, 12 , and 16.

The maximal value of the vorticity $L_{\max }$ and the $S / N$ transition fields for the five different topologies are summarized in Table. I. There is a substantial increase of $L_{\max }$ when an antidot is introduced in the square sample. $L_{\max }$ is practically independent of the number of antidots. For the twoantidot sample $L_{\max }=18$ is one unit smaller, which may be the consequence of the fact that only even $L$ can nucleate as a ground state in the high magnetic-field region. The $S / N$ critical field increases only by $3.4 \%$ when we go from the one- to the four-antidot sample. This indicates that the size of the narrowest superconducting area in the sample mainly determines the $S / H$ transition field.

Figure 3 shows the magnetization of the different vortex states for the same samples as considered in Fig. 2, i.e., for the full square (a) and squares with one (b), two (c,d), three

TABLE I. Maximum vorticity $L_{\max }$ and the $S / N$ transition field for the five different superconducting structures depicted in Fig. 1

\begin{tabular}{lcc}
\hline \hline & $L_{\max }$ & $H_{c 3} / H_{c 2}$ \\
\hline Full square & 11 & 2.01 \\
One antidot & 19 & 3.21 \\
Two diagonal antidots & 18 & 3.22 \\
Two top antidots & 19 & 3.28 \\
Three antidots & 19 & 3.30 \\
Four antidots & 19 & 3.32 \\
\hline \hline
\end{tabular}




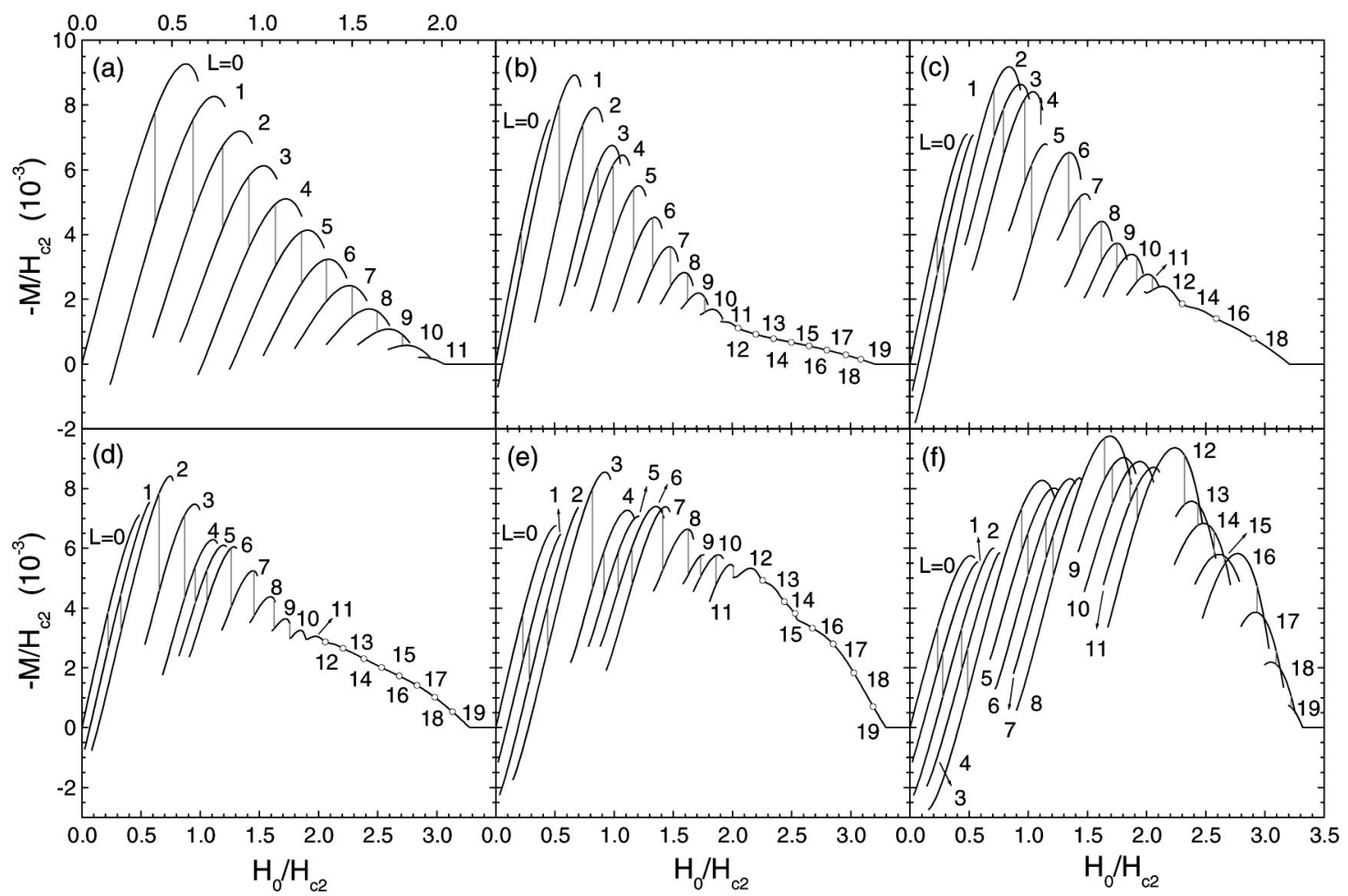

FIG. 3. The magnetization as a function of the applied magnetic field for the reference sample (a) and for the square with one (b), two diagonal (c), two top (d), three (e) and four (f) holes. The vertical lines show the ground-state transitions between different vortex states, and open circles indicate continuous transitions between different vortex states.

(e), and four (f) holes. The magnetization is a measure of the expelled flux from the sample and is calculated after averaging the field only over the superconducting region excluding the holes. In these figures the vertical gray lines indicate the ground-state transitions. Notice that in the rest of the text we define the magnetization as $-M$, i.e., the difference between the applied magnetic field and the averaged magnetic field.

The magnetization curves are strongly influenced by the presence of the antidots. In the absence of the antidots [see Fig. 3(a)] the maxima in the magnetization curve decreases with increasing $L$ which is not so for the other samples. For the single-antidot sample the largest flux expulsion is reached for $L=1$, while this is realized in the case of the two- (three-) antidot sample for $L=2$ (3), i.e., it equals the number of antidots. The magnetization also depends on the arrangement of antidots in the sample. For example, the magnetization of the states with $1<L<11$ for the sample with two antidots along the diagonal [Fig. 3(c)] is higher than the magnetization for the sample with two antidots on the top row [Fig. 3(d)], while for the other vortex states it is opposite. The sample with four antidots behaves very different and we found that $-M$ is maximal for $L=8$. There is also a clear bunching of the magnetization curves with increasing the number of antidots which is absent in our reference sample. The number of curves that are bunched together increases with the number of antidots. The Meissner state, i.e., $L=0$ state becomes less stable when antidots are present. The transition to the $L=1$ state occurs at a lower magnetic field indicating that the presence of the antidot(s) eases the penetration of the first vortex. For the supercon- ductors with one (b), two (c,d), and three (e) holes the lowest magnetization is reached for the state where $L$ equals the number of holes, i.e., in that case the largest paramagnetic response (i.e., $-M<0$ ) can be realized. Only the vortex states with vorticity less or equal to the number of antidots exhibit a paramagnetic response. Note that for the reference sample a paramagnetic response $\mathrm{e}^{35}$ can be realized in a small magnetic-field region of the $L=1,4,5$ states. It should be stressed that the superconductor is in a metastable state when such a paramagnetic response is found. Notice also the difference in the magnetization for the different two-antidot samples.

In experiment one measures the magnetization by averaging the magnetic field over some area, which is determined by the size of the detector. In the case of samples with antidots, the magnetic-field distribution is extremely nonuniform inside as well as outside the sample and therefore the detector size will have an effect on the measured magnetization. To illustrate this we calculated the magnetization by averaging the magnetic field over the area $W \times W$. As an example, we plotted the magnetization for the four-antidot sample in Fig. 4, which exhibits a quite different behavior. The magnetization does not decrease monotonically by increasing $L$, but it is periodical. Now the maximal magnetization is found for the states with $L=0$ and $L=4$. The states are now clearly bunched together in groups of four. Notice also that many vortex states exhibit paramagnetic response, which clearly is very sensitive to the definition of $M$ and that there are ground-state transitions to the states with $-M<0$ !

Figure 5 shows the magnetic-field range $\Delta H_{s}$ over which 


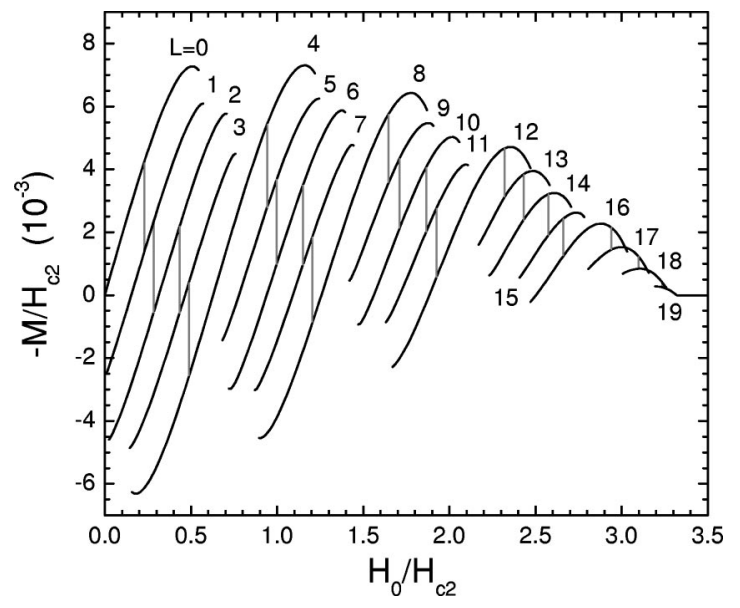

FIG. 4. The magnetization of the four-antidot sample as a function of the applied magnetic field when the field is averaged over the area $W \times W$.

the vortex state with vorticity $L$ is stable (i.e., $\Delta H_{s}$ $=H_{\text {penetration }}-H_{\text {expulsion }}$ ) as a function of the vorticity $L$. For the reference sample the result is shown by the full circles, for the one-antidot sample by the open circles, for the two-diagonal-antidot sample by the full triangles, for the two-top-antidot sample by the open triangles, for the threeantidot sample by the full squares and for the four-antidot sample by the open squares. The results for the two-diagonal, two-top, three- and four-antidot samples are shifted over $0.2 / H_{c 2}, 0.5 / H_{c 2}, 0.6 / H_{c 2}$, and $0.8 / H_{c 2}$, respectively. The stability of each individual superconducting state is very sensitive to the topology of the sample. For the reference sample $\Delta H_{s}(L)$ decreases with increasing $L$, except for the $L=4$ state which exhibits an enhanced stability and which is

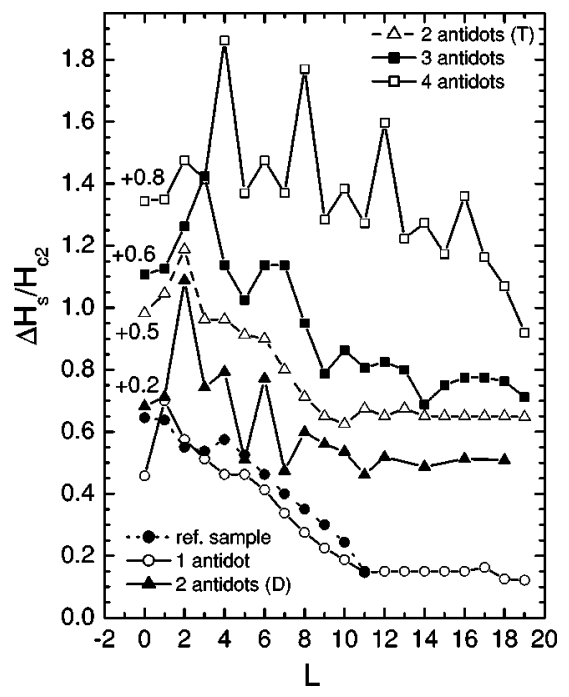

FIG. 5. The magnetic-field region $\Delta H_{s}$ over which the state with vorticity $L$ is stable as a function of the vorticity $L$, for the reference sample (full circles), for the sample with one (open circles), two diagonal (full triangles), two-top (open triangles), three (full squares) and four (open square) holes. The result for twodiagonal, two-top, three- and four-antidot samples are shifted over $0.2 H_{c 2}, 0.5 H_{c 2}, 0.6 H_{c 2}$, and $0.8 H_{c 2}$, respectively.

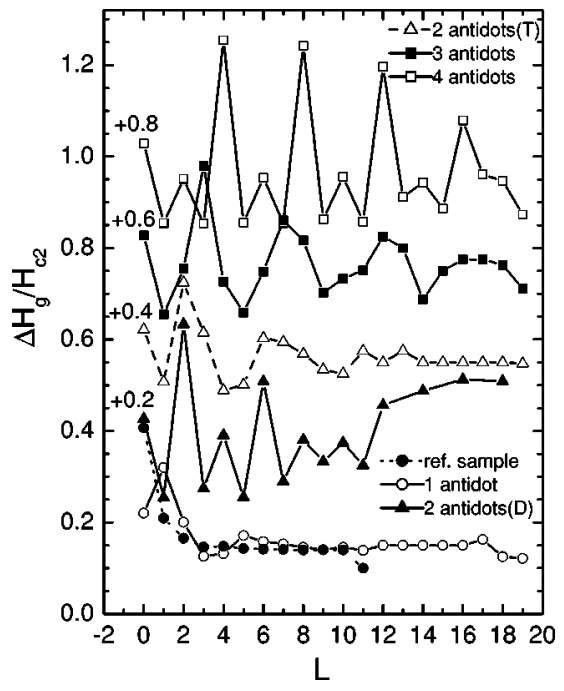

FIG. 6. The magnetic-field region $\Delta H_{g}$ over which the state with vorticity $L$ is the ground state as a function of the vorticity $L$, for the reference sample (full circles), for the sample with one (open circles), two-diagonal (full triangles), two-top (open triangles), three (full squares), and four (open square) holes. The result for two diagonal, two top, three- and four-antidot samples are shifted over $0.2 \mathrm{H}_{c 2}, 0.4 \mathrm{H}_{c 2}, 0.6 \mathrm{H}_{c 2}$ and $0.8 \mathrm{H}_{c 2}$, respectively.

clearly a consequence of the commensurability of the square vortex lattice with the square geometry of the sample as we pointed out earlier. ${ }^{32}$ For the square with a single hole, the state with $L=1$ has the largest stability range. With increasing vorticity, the stability region decreases monotonically, except for the state with $L=5$ and 17. Notice that (1) for $L$ $>2$ the different vortex states in the reference sample have a large stability region than those for the sample with one antidot and (2) for $L \geqslant 11$ the stability region is practically independent of $L$, i.e., it is the magnetic field region where all $L$ transitions are continuous. For the superconductors with two-diagonal and four holes, the vortex states with even vorticity are more stable than those with odd vorticity. This is less pronounced in the case of the two top antidot sample. For the four-antidot system vortex states in which $L$ are multiples of 4 have the highest stability. But for the superconductor with three holes the $L=3$ and $L=6,7$ states have a substantial larger stability region as compared to the other vortex states. The $L$ states with enhanced stability are clearly a consequence of matching phenomena.

The magnetic-field range over which each of the vortex states is the ground state $\Delta H_{g}$ is shown in Fig. 6 for the different geometries as a function of the vorticity $L$. The ground-state region $\Delta H_{g}$ also shows similar features as the stability region $\Delta H_{s}$. There is one main difference, $\Delta H_{s}$ exhibits an overall decrease with increasing $L$ in the larger magnetic-field region which is not present in $\Delta H_{g}$.

In all cases, the vortex states show enhanced stability for commensurate vorticity, namely, when the number of vortices penetrating the sample is a multiple of the number of holes. However, for higher magnetic field these commensurability effects disappear or are less pronounced, which is due to the finite size of the sample. 


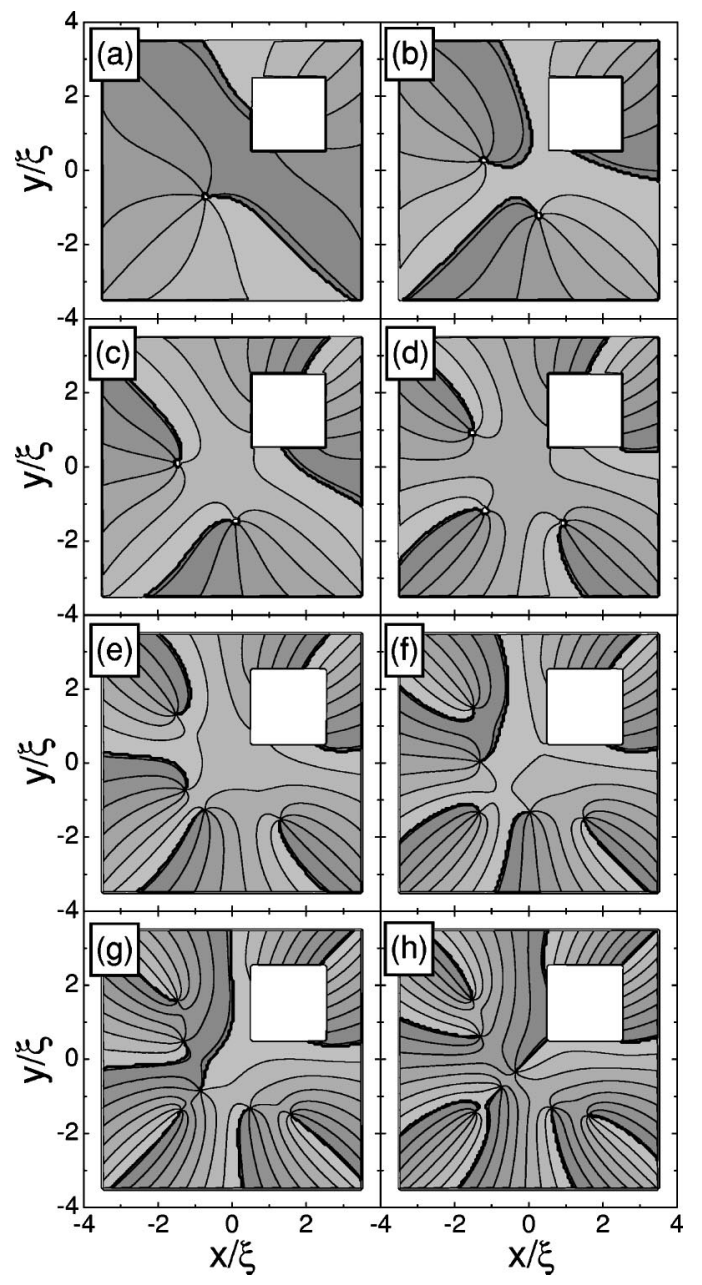

FIG. 7. The phase of the order parameter for the superconductor with one hole for the states with $L=2$ (a), 3 (b), 4 (c), 5 (d), 6 (e), 7 (f), $8(\mathrm{~g})$, and $9(\mathrm{~h})$ at $H_{0} / H_{c 2}=0.62,0.82,0.92,1.07,1.22,1.37$, 1.52 , and 1.67 , respectively. Phases near zero are given by light gray regions, phases near $2 \pi$ by dark gray regions.

\section{SPATIAL DISTRIBUTION OF THE VORTICES}

In the full square sample, vortices may form multivortex states with vorticity $L=2,3,4,5$, and 6 . By further increasing the magnetic field, vortices move towards the center and form a giant vortex state (see also Ref. 32).

When one antidot is placed in the upper right half of the sample, the square symmetry is broken and the hole acts like a pinning center. As a consequence the multivortex state will become more favorable and stay stable even up to higher fields and vorticity. For the multivortex states with higher vorticity, the separate multivortices are not visible anymore in the contour plots of the magnetic-field distribution or Cooper-pair density. The reason is that the vortices are too close to each other, the size of the Cooper-pair density is very small, and the spots corresponding to high magnetic fields are overlapping. Therefore to visualize the vortex configurations we plot the phase of the order parameter for the sample with one hole in Figs. 7(a) $-7(\mathrm{~h})$ for the states with $L=2-9$ at $H_{0} / H_{c 2}=0.62,0.82,0.92,1.07,1.22,1.37$, $1.52,1.67$, respectively. All states correspond to ground

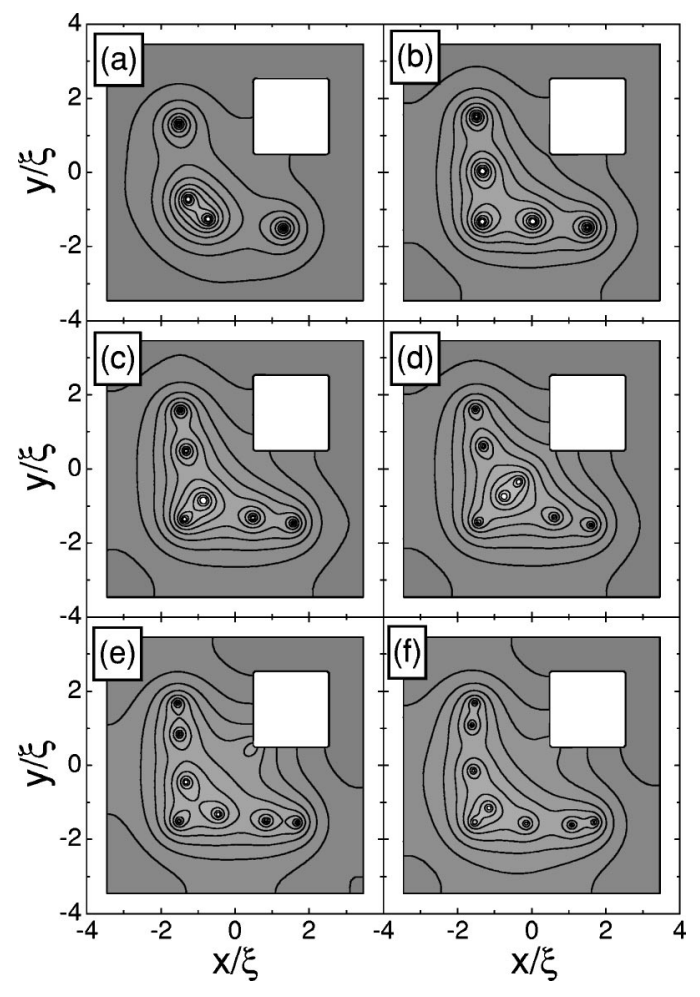

FIG. 8. Contour plot of the Cooper-pair density in logarithmic scale for the one-antidot sample corresponding to the $L=6$ (a), 7 (b), 8 (c), 9 (d), 10 (e), and 11 (f) states at $H_{0} / H_{c 2}=1.22,1.37$, $1.52,1.67,1.82$, and 1.95, respectively. Dark (light) gray regions correspond to high (low) density. The scales are (a) $3 \times 10^{-4}-0.9$, (b) $3 \times 10^{-4}-0.9$, (c) $8 \times 10^{-5}-0.9$, (d) $1 \times 10^{-5}-0.8$, (e) 1 $\times 10^{-5}-0.8$, and (f) $1 \times 10^{-6}-0.8$.

states at the given magnetic fields. In these figures phases near zero are given by light gray regions and phases near $2 \pi$ by dark gray regions. In order to determine the number of vortices in some region, we should "go" around this region. If the vorticity in this region is $L$, then the phase changes $L$ times $2 \pi(\Delta \varphi=2 \pi L)$.

In the one-antidot sample the first vortex will sit in the hole. We found that the second vortex is located in the superconducting material on the same diagonal where the hole is located [Fig. 7(a)]. The third vortex nucleates in the superconducting material and pushes the second vortex away from the diagonal [Fig. 7(b)]. By increasing the magnetic field these vortices move towards the hole. The fourth vortex is pushed inside the hole, where now two vortices are located [Fig. 7(c)]. The fifth vortex is again nicely located on the diagonal [Fig. 7(d)]. The sixth vortex is located in the superconducting region, where two vortices are located very close to each other in the opposite corner of the antidot [Fig. 7(e)]. The latter two vortices form almost a giant vortex state, but they can be resolved as two separate vortices if we plot the phase of $\Psi$ [Fig. 7(e)] or if we make a log-scale contour plot of the Cooper-pair density [see Fig. 8(a)]. The Cooper-pair density variation between these two vortices is of order 3 $\times 10^{-4}-7 \times 10^{-4}$ and, therefore one will probably not be able to resolve them experimentally as two separate vortices. Increasing the vorticity to $L=7$ the latter two vortices move 
apart and an additional vortex is added forming a local triangular arrangement [see Figs. 7(f) and 8(b)]. For the states with $L=8$ [Fig. 7(g)] and $L=9$ [Fig. 7(h)], the number of vortices in the hole is unaltered and additional vortices are added along the diagonal passing through the antidot [Figs. 8(c,d)]. Increasing the field further to $L=10$ [see Fig. 8(e) for $\left.H_{0} / H_{c 2}=1.82\right]$ an additional vortex enters the antidot where now three vortices are located. The vortex arrangement for the $L=11$ state with $H_{0} / H_{c 2}=1.95$ is shown in Fig. 8(f). For the $L=16$ state four vortices are inside the hole which is the maximum number of vortices that can be contained in the hole. As is apparent from Figs. 7 and 8 the vorticity of particular vortices in the one-hole sample may vary, but the distribution of vortices in the superconducting region is always symmetric with respect to the diagonal passing through the hole. From Fig. 8 we clearly see that the variation in the Cooper-pair density between the vortices is very small and as a consequence we expect that experimentally the multivortex area will be observed rather as a triangularlike shaped giant vortex state.

The sample with two antidots [see Fig. 1(c,d)] is more symmetric, which will have an influence on the position of the vortices. We show in Figs. 9(a) $-9(\mathrm{~h})$ the phase of the order parameter for the states with vorticity $L=3-10$ at $H_{0} / H_{c 2}=0.76,0.87,1.02,1.1,1.38,1.51,1.67,1.81$, respectively. At these magnetic fields the vortex states in question correspond to the ground state. The $L=1$ and $L=2$ vortex configurations are trivial. For $L=1$ there is one vortex in one of the antidots and the ground state is degenerate with respect to which antidot the vortex is located. This degeneracy is a new aspect which was not present in previous sample. When $L=2$ each antidot contains a single vortex. For $L=3$ the extra vortex is located in one of the antidots [Fig. 9(a)] and the superconducting state is again degenerate. When $L=4$ two vortices nucleate in each of the antidots [Fig. 9(b)]. The fifth and the sixth vortex are situated in the superconducting region along the diagonal [Figs. 9(c,d)]. When the seventh vortex enters the superconductor, its position is close to the center of the sample [Fig. 9(e)] and the state is degenerate with a similar vortex configuration in which the additional vortex is to the right side of the diagonal passing through the two antidots. This nonsymmetric vortex arrangement with respect to the diagonal passing through the antidots is energetically preferred because of the narrow superconducting region between the two antidots where superconductivity is enhanced ${ }^{34}$ Figure 9(f) shows that for $L$ $=8$ there are two vortices in each hole and the other vortices are along the diagonal in the superconducting region forming two clusters each consisting of two closely spaced vortices. The ninth vortex is stabilized in the center of the sample [Fig. $9(\mathrm{~g})]$. When the tenth vortex enters the superconductor, it initially forms a giant vortex in the center with vorticity $L=2$, but we find that this giant vortex is not stable and eventually the giant vortex decays in two separate vortices, each of them moves to each of the holes [Fig. 9(h)]. Till the $L=16$ state all other vortices are situated along the diagonal. Increasing $L$ further we found that the number of vortices in each hole increases with one unit. Superconductivity is then only preserved in the corners of the sample where the holes

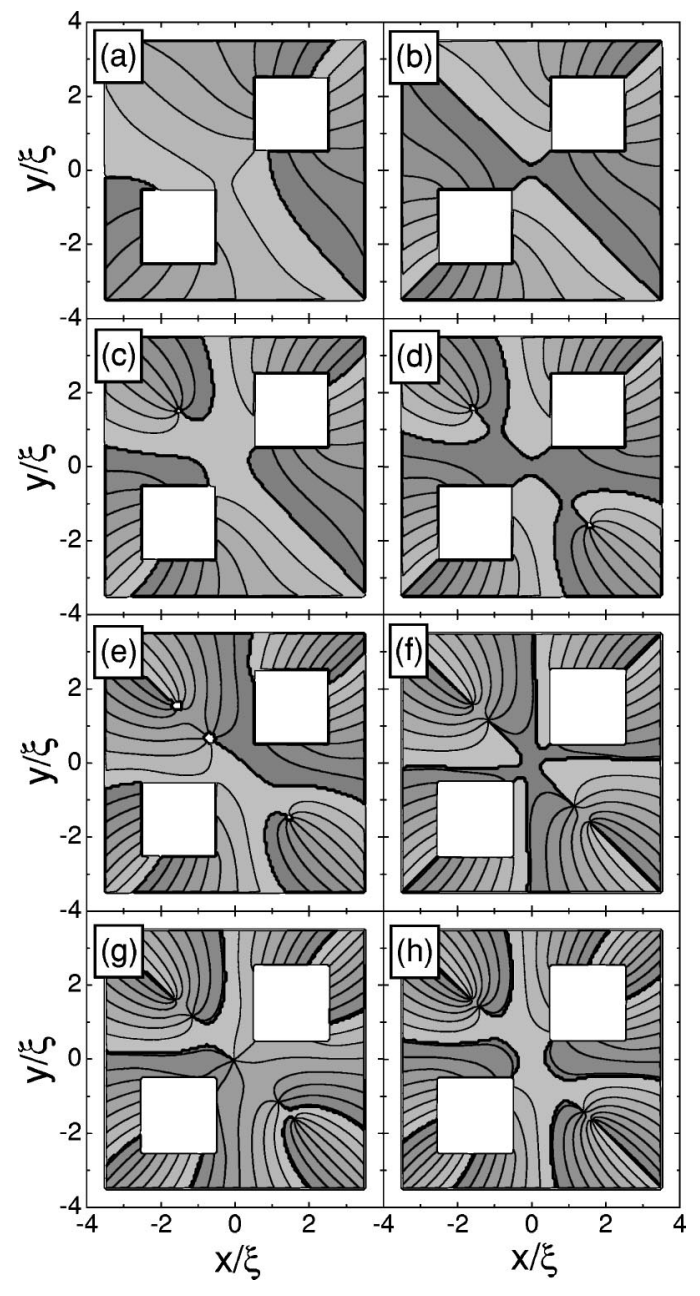

FIG. 9. The phase of the order parameter for the superconductor with two-diagonal holes for the states with $L=3$ (a), 4 (b), 5 (c), 6 (d), 7 (e), 8 (f), 9 (g), and $10(\mathrm{~h})$ at $H_{0} / H_{c 2}=0.76,0.87,1.02,1.1$, $1.38,1.51,1.67$, and 1.81 , respectively. Phases near zero are given by light gray regions, phases near $2 \pi$ by dark gray regions.

are placed and the rest of the sample is practically in the normal state.

In order to show the influence of the symmetry we plotted the phase of the order parameter for the sample with two antidots, where the antidots are at the upper half of the sample [Figs. 10(a)-10(h)]. The first and the second vortex are, as expected, in the holes. The third vortex is located in the superconducting region [Fig. 10(a)]. For the $L=4$ state two vortices in the superconducting region are located symmetrically [Fig. 10(b)] and this symmetry is broken when the fifth vortex enters the hole [Fig. 10(c)]. Up to the $L=10$ state [Figs. $10(\mathrm{~d})-10(\mathrm{~g})]$ two vortices are in each hole and the other vortices are in the superconducting region, forming different vortex configurations. At the lower magnetic-field region for the $L=10$ state, each hole contains two vortices and the other two vortices are situated close to the holes [Fig. 10(h)]. By further increasing the field these two vortices eventually enter the holes.

The symmetry is reduced when we add a third antidot [Fig. 1(e)]. In Figs. 11(a)-11(h) the phase of the order parameter for the superconductor with three holes is given for 


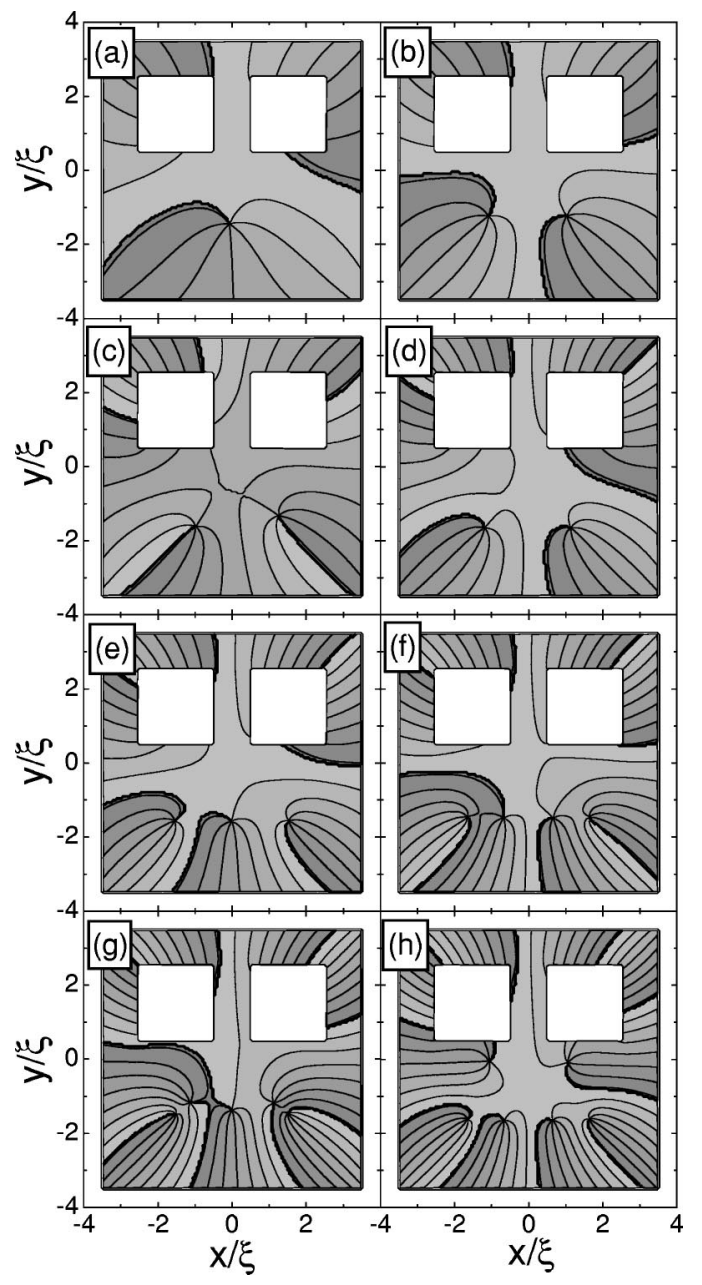

FIG. 10. The phase of the order parameter for the superconductor with two top holes for the states with $L=3$ (a), 4 (b), 5 (c), 6 (d), 7 (e), 8 (f), 9 (g), and $10(\mathrm{~h})$ at $H_{0} / H_{c 2}=0.67,0.9,0.97,1.07$, $1.27,1.47,1.65$, and 1.77 , respectively. Phases near zero are given by light gray regions, phases near $2 \pi$ by dark gray regions.

the states with $L=4-11$ at $H_{0} / H_{c 2}=0.87,0.97,1.02,1.195$, $1.47,1.65,1.77,1.92$, respectively. In this case the first vortex is located in one of the two antidots, situated along the diagonal of the sample. The second vortex is then placed in the opposite antidot along the diagonal. For $L=3$ each antidot contains a single vortex and the vortex configuration is very stable (see Figs. 5 and 6). The fourth vortex enters into the superconducting region [Fig. 11(a)]. The next vortex nucleates in the upper antidot of the sample [Fig. 11(b)]. For $L=6$ the two antidots along the same diagonal contain each two vortices [Fig. 11(c)]. In the $L=7$ state there are two vortices in each of the holes [Fig. 11(d)]. The eighth vortex enters into the superconducting region [Fig. 11(e)] and forms a tight cluster of two vortices which is practically a $L=2$ giant vortex. For the vortex state with $L=9$, each antidot contains two vortices and three separate vortices are located in the superconducting region [Fig. 11(f)] in a triangle arrangement. When the next vortex enters the sample it goes to the upper hole and one of the vortices from the superconducting region moves to the bottom right hole [Fig. 11(g)]. When there are 11 vortices in the sample [Fig. 11(h)], each

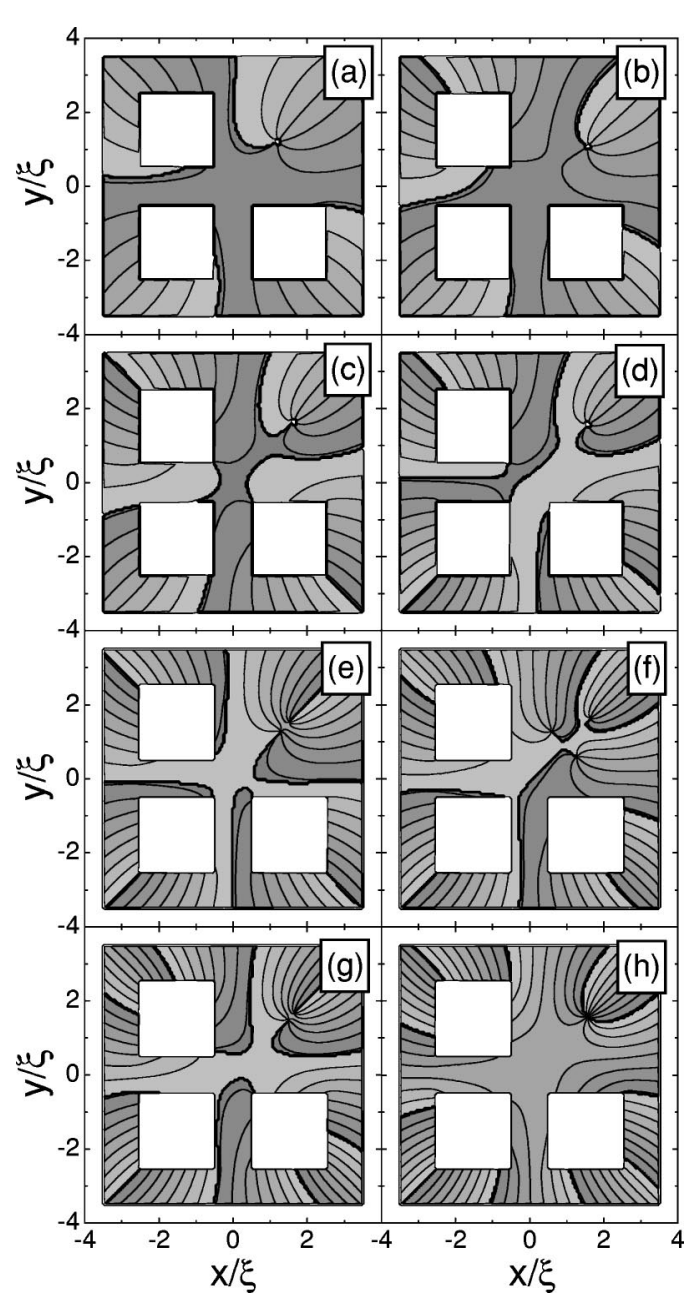

FIG. 11. The phase of the order parameter for the superconductor with three holes for the vortex states with $L=4$ (a), 5 (b), 6 (c), 7 (d), 8 (e), 9 (f), 10 (g), and 11 (h) at $H_{0} / H_{c 2}=0.87,0.97$, $1.02,1.195,1.47,1.65,1.77$, and 1.92, respectively. Phases near zero are given by light gray regions, phases near $2 \pi$ by dark gray regions.

of the antidots contains three vortices and one giant vortex with $L=2$ nucleates in the superconducting region. Until the $L=15$ state each antidot contains three vortices and the other vortices are located in the superconducting region. Increasing the field changes the vortex arrangement in the superconducting region. For example, for $L=12$ three vortices are located along the diagonal and increasing the field rearranges them such that they form a triangle lattice. For $L=15$ the fourth vortex enters the lower left hole. For the $L=16$ state each hole contains four vortices and until the superconductor transforms to the normal state no more vortices are added to the antidots.

For the sample with four antidots [Fig. 1(f)] the vortices are, up to high magnetic fields, located in the antidots. As a consequence the phase of the superconducting condensate $\Psi$ contains less information and therefore we show in Figs. 12(a)-12(h) the magnetic-field distribution for the sample with four holes for the states with vorticity $L=1-4,12,13$, 14 , and 17 at $H_{0} / H_{c 2}=0.25,0.33,0.46,0.67,1.2,2.36$, 2.48 , and 2.97, respectively. At these values of the magnetic 


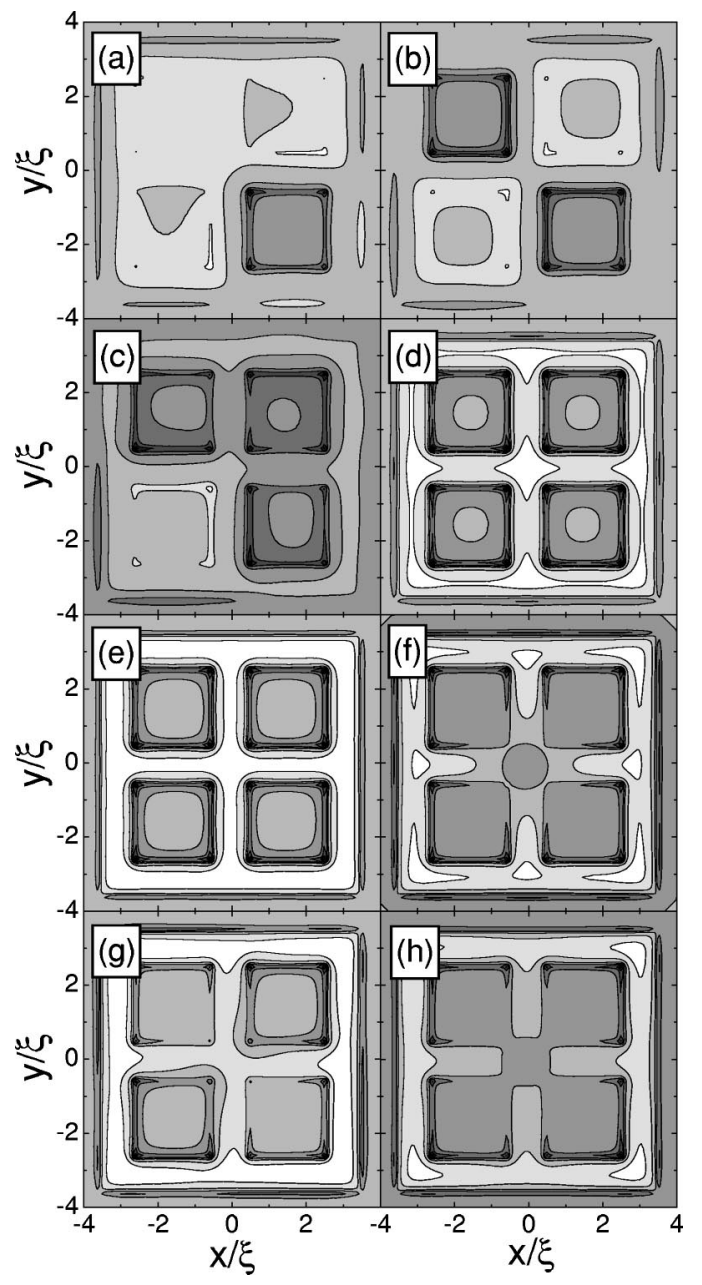

FIG. 12. The magnetic-field distribution for the superconductor with four holes for the states with vorticity $L=1$ (a), 2 (b), 3 (c), 4 (d), 12 (e), 13 (f), $14(\mathrm{~g})$, and 17 (h) at $H_{0} / H_{c 2}=0.25,0.33,0.46$, $0.67,1.2,2.36,2.48$, and 2.97, respectively. Higher magnetic field is given by dark gray regions and lower by light gray regions.

fields, the considered states correspond to the ground state of the superconductor. The applied magnetic field is always given by the same gray color. An increase (decrease) of the local field with respect to the applied field is indicated by a darker (lighter) color. Up to the first penetration field, the magnetic field is expelled from the superconductor and we see an increased magnetic field near the boundary of the superconductor. But even in this case there is some increased magnetic field in all antidots. At $H_{0} / H_{c 2}=0.56$ the first vortex enters the superconductor. One can expect that the position of this vortex can be in any of the four holes [Fig. 12(a)] (for a much smaller size of the superconductor this vortex can be located in the center of the superconductor). The second vortex is situated in the hole opposite to the hole containing the first vortex [Fig. 12(b)]. The probability for the third vortex to be located in one of the remaining two holes is equal. In the depicted configuration the third vortex is in the top right hole, as shown in Fig. 12(c). For the $L=4$ state all antidots contain a single vortex [Fig. 12(d)]. This rule of filling the antidots with vortices is repeated up to the 12th vortex [Fig. 12(e)]. The 13th vortex appears in the center of

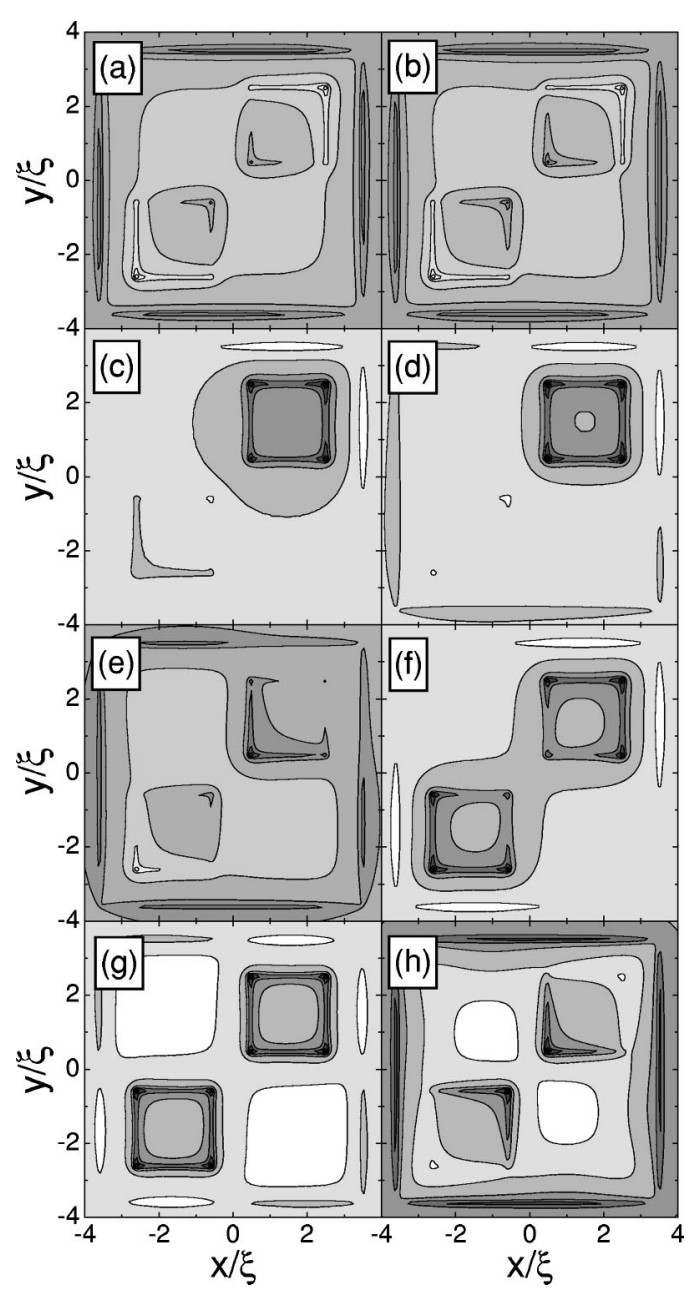

FIG. 13. The magnetic-field distribution for the superconductor with two holes for the states with vorticity $L=0$ at $H_{0} / H_{c 2}=0.02$ (a) and 0.47 (b), with vorticity $L=1$ at $H_{0} / H_{c 2}=0.03$ (c), 0.13 (d) and 0.53 (e) and with vorticity $L=2$ at $H_{0} / H_{c 2}=0.08$ (f), $0.28(\mathrm{~g})$, and 0.92 (h). Dark gray regions correspond to high magnetic field.

the sample [Fig. 12(f)], which is due to the small sizes of the holes that prevent them from capturing more vortices at those fields and the fact that if the extra vortex would go to one of the antidots a very asymmetric configurations would be obtained, which is energetically unfavored. When the 14th vortex appears in the superconductor, the symmetry can be restored by moving two vortices to the holes [Fig. 12(g)]. Starting from $L=17$, the superconductivity in the central region of the sample is destroyed and vortices move to the center. After the 19th vortex the sample transforms into the normal state.

The magnetic-field distribution in the superconductor and inside the antidots is also of interest. As an example, we consider the magnetic-field distribution (Fig. 13) in the superconductor with two antidots for vorticity $L=0,1$, and 2, where the value of the magnetic field is taken such that it corresponds to the ground state. The dark regions in the figures correspond to high magnetic fields. It is clear from these figures that the magnetic field is nonuniform in and around the sample and inside the antidots. For low magnetic fields the superconductor expels the magnetic-field and the mag- 
netic field lines are bent around the superconductor, which leads to, so-called, demagnetization effects. Figure 13(a) shows the magnetic-field distribution for the state with vorticity $L=0$ (we know this, e.g., from the phase of the order parameter). Even in this case there is a small increase of the magnetic field inside both holes. With increasing external field the intensity of the field in the holes also increases [Fig. 13(b)]. Outside the antidots the magnetic field is expelled towards the holes and consequently near, but inside the holes there is a higher density of magnetic field lines. Starting from $H_{0} / H_{c 2}=0.03$ the first vortex enters the superconductor and a single vortex is pinned by one of the antidots [Fig. 13(c)]. The distribution of the magnetic field in the hole is also nonuniform. The magnetic field is lower in the central part of the hole and peaks in the magnetic-field strength are found near the four corners of the antidot. With increasing field the vortex is compressed towards the center of the superconductor [Figs. 13(d,e)]. Further increase of the field beyond $H_{0} / H_{c 2}=0.08$ leads to the appearance of a vortex in the second hole [Fig. 13(f)]. In this case the intensity of the magnetic field in the first hole decreases. At the smaller field region the intensity of the field in both holes is higher near the corners of the superconductor. With increasing external field vortices move toward the center of the superconductor [Figs. 13(g,h)]. It is clear that with increasing external field and fixed number of vortices the demagnetization effects are more pronounced, because the superconductor has to expel more magnetic field.

As is well known, when a superconducting sample is placed in an external magnetic field, the magnetic field is expelled from the superconductor due to screening currents near the sample boundary. The direction of the screening current is such that the corresponding created magnetic field is opposite to the external one, which leads to a lower total field inside the superconductor. Magnetic field, i.e., vortices, penetrating the superconductor creates currents flowing in the opposite direction to the screening currents. The competition between these currents results in the creation of vortices.

Examples of vector plots of the current density are shown in Fig. 14. In Figs. 14(a)-14(e) we show the results for the superconductor with four antidots for the $L=0-4$ states at $H_{0} / H_{c 2}=0.07,0.57,0.6,0.75,0.78$, respectively, and Figs. 14(f) -14 (h) show vector plots of the current density in the sample with two antidots for the states with $L=0-2$ at $H_{0} / H_{c 2}=0.07,0.52,0.57$, respectively. For convenience we labeled the holes.

Let us first consider the current distribution for the superconductor with four holes. In the zero-vorticity state the screening currents near the sample boundary flow clockwise [Fig. 14(a)]. The magnetic field is expelled from the whole sample, including the antidots. The intensity of the currents in the center is lower than the intensity of the currents near the outer edge. Thus around the holes we see that near the outer boundaries the current flows clockwise, while at the inner boundaries the current flows counterclockwise. In Fig. 14(b) the current distribution is shown for the state with vorticity $L=1$. The vortex is located in the "fourth" hole around which the current flow in the counterclockwise direc-

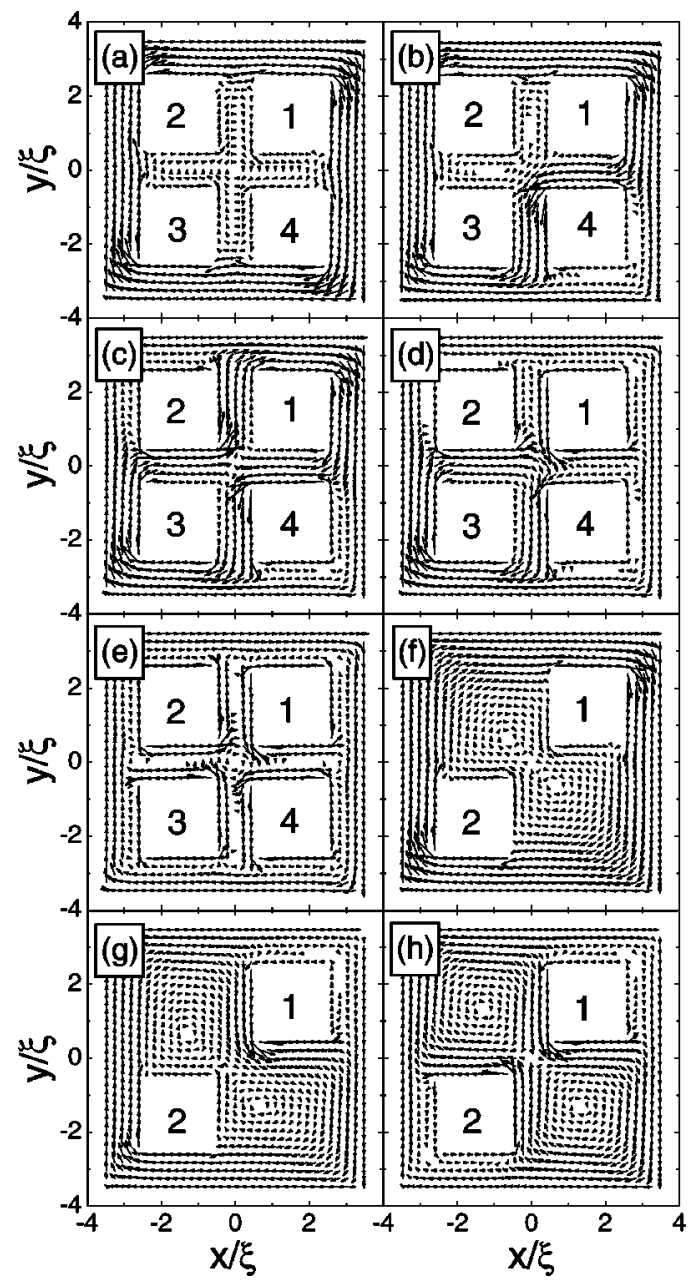

FIG. 14. Vector plots of the supercurrent in the superconductor with four holes for the vortex states with $L=0$ (a), 1 (b), 2 (c), 3 (d), and 4 (e) at $H_{0} / H_{c 2}=0.07,0.57,0.56,0.75$, and 0.78 , respectively, and in the superconductor with two holes for the states with $L=0$ (f), $1(\mathrm{~g})$, and $2(\mathrm{~h})$ at $H_{0} / H_{c 2}=0.07,0.52$, and 0.57 , respectively.

tion. The current flows around the edge of the sample and around the other antidots are the same as in Fig. 12(a). When the second vortex enters the superconductor [Fig. 14(c)], the extra vortex appears in the second hole and the currents around the first and the second hole flow counterclockwise, which is responsible for an increase of the magnetic field in the antidots 2 and 4, while around the antidots 1 and 3 a clockwise direction of the current is seen which expels the field inside these antidots. Note also that, as the value of the magnetic field is larger than in the previous figures, the intensity of the current is also larger. In the state with three vortices [Fig. 14(d)] the current also flows counterclockwise around the first hole, where the third vortex is situated [see Fig. 12(c)]. We can see a decrease of the intensity of the current in the corners near the holes 1 and 2. From the vector plot of the current density one can expect antivortices in the center and near the boundary of the sample, because there are some spots where the currents flow in the clockwise direction, while currents around the holes flow counterclockwise [Fig. 14(e)]. This occurs because of the cancellation 


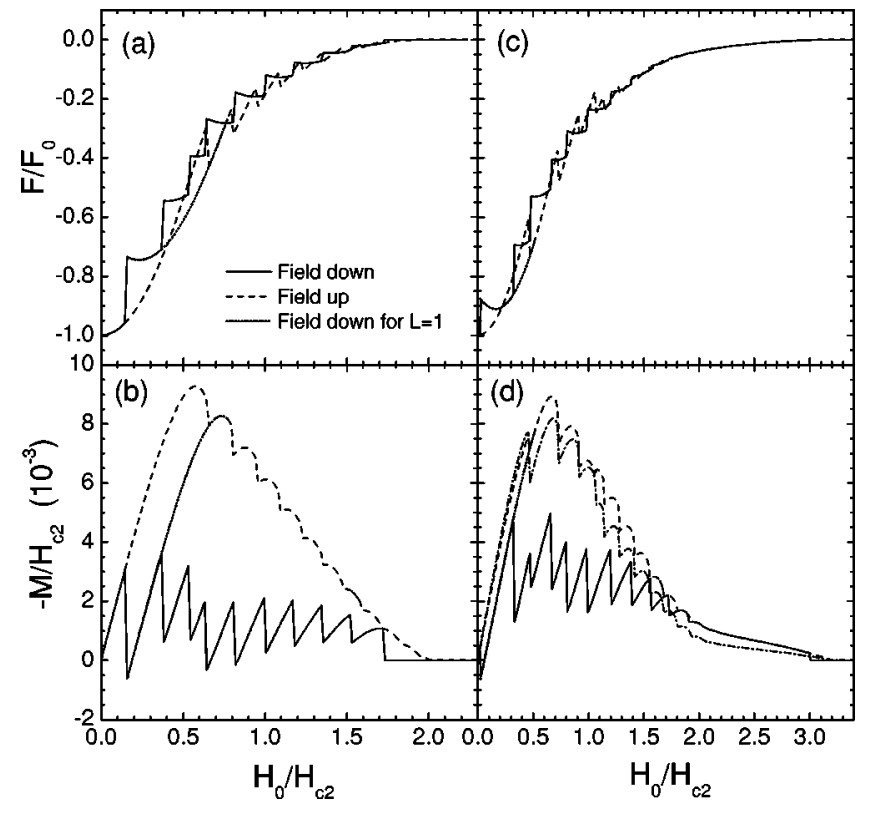

FIG. 15. The free energy and magnetization as a function of the applied magnetic field for the reference superconductor $(\mathrm{a}, \mathrm{b})$ and for the sample with a hole $(\mathrm{c}, \mathrm{d})$. Dashed curves correspond to the case magnetic field sweep up, solid curves correspond to the case magnetic field sweep down and dotted curves correspond to the field sweep down for the $L=1$ state. The dash-dotted curve in (d) shows the magnetization when the field is averaged over the area $W \times W$ for increasing field.

between the screening currents and the currents around the holes, which do not lead to antivortices (we checked this from the phase of the order parameter).

For the sample with two holes, the current flows clockwise in the whole superconductor in the Meissner state [Fig. 14(f)]. The current can be decomposed in a clockwise flow near the perimeter and a clockwise flow in the shape of a noncrossing eight across the upper left and right bottom region. When the first vortex "appears" [Fig. 14(g)], the vortex is in hole 1 and the current flowing around antidot 1 cancels the screening current near the corner of the superconductor, which leads to a weakening of superconductivity in this region. The current distribution in the other parts of the superconductor is qualitatively not changed. For the state with two vortices the current flows counterclockwise around both holes [Fig. 14(h)] as expected.

\section{TRANSITION BETWEEN VORTEX STATES}

In order to investigate the vortex configuration in more detail we studied our samples as a function of a decreasing and an increasing magnetic field. In doing so we can investigate vortex expulsion and penetration into the superconductor.

Figure 15 shows the free energy and magnetization as functions of the applied magnetic field for the reference sample $(\mathrm{a}, \mathrm{b})$ and for the sample containing a single hole $(c, d)$. The dashed curve corresponds to the case for increasing magnetic field, the solid curve corresponds to a field sweep down, and the dotted curve is for a field sweep up and

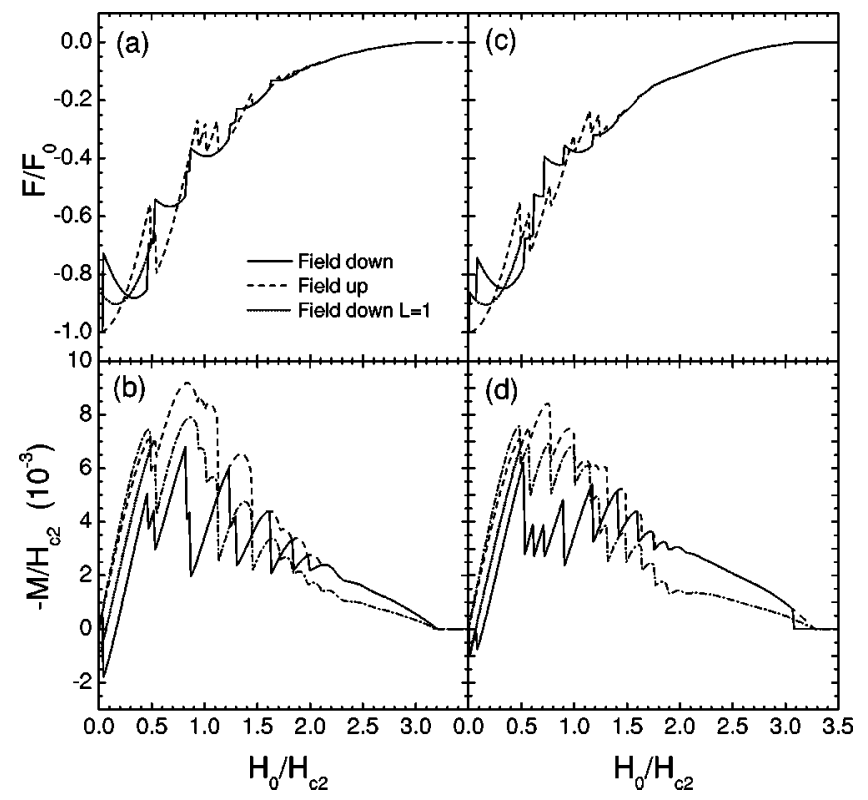

FIG. 16. The free energy and magnetization as functions of the applied magnetic field for the superconductors with two-diagonal $(\mathrm{a}, \mathrm{b})$ and with two-top (c,d) holes. Solid curves correspond to the case magnetic field sweep down and dashed curves correspond to the case magnetic field sweep up. Dotted curves indicate the $L=1$ state. The dash-dotted curves correspond to the magnetization when the field is averaged over the area $W \times W$ for increasing field.

down when the superconductor is locked into the $L=1$ state. In decreasing field the reference sample drops to the superconducting state with nine vortices. Decreasing further the field we find all $L \rightarrow L-1$ transitions. If we decrease the field beyond zero to negative fields we obtain a free energy which is symmetrical with respect to the curves we obtained for increasing field. In the case a single hole is present [Figs. $15(\mathrm{c}, \mathrm{d})]$ we find continuous transitions between vortex states up to the $L=10$ state. All other transitions are $L \rightarrow L-1$ transitions and occur with a jump in the free energy. Notice that (1) on average the magnetization for the one-antidot sample, calculated averaging the field only over the superconducting region (dashed curve), is larger than for our reference sample and (2) beyond $H_{0} / H_{c 2}>1.7$ both magnetization curves are markedly different. We also calculated the magnetization for the one-antidot sample averaging the magnetic field over the $W \times W$ region [Fig. 15(d) dash-dotted curve], which shows similar features as the magnetization of only superconducting region [Fig. 15(d) dashed curve]. In this case the magnetization is lower for all $L$ states, except the $L=0$ state.

Figures 16(a)-16(d) show the free energy and magnetization as a function of applied magnetic field for a field sweep up (dashed curves) and field sweep down (solid curves) for the samples with two antidots along the diagonal $(a, b)$ and the one with the antidots along the top row $(\mathrm{c}, \mathrm{d})$ of the sample. In the case of the superconductor with two-diagonal holes we can see transitions between states with $L=4$ to $L$ $=6$, with $L=6$ to $L=8$, with $L=12$ to $L=14$, and with $L$ $=14$ to $L=16$ for field sweep up [Fig. 16(a)]. For the field sweep down we see all $L \rightarrow L-1$ transitions, except the $L$ 


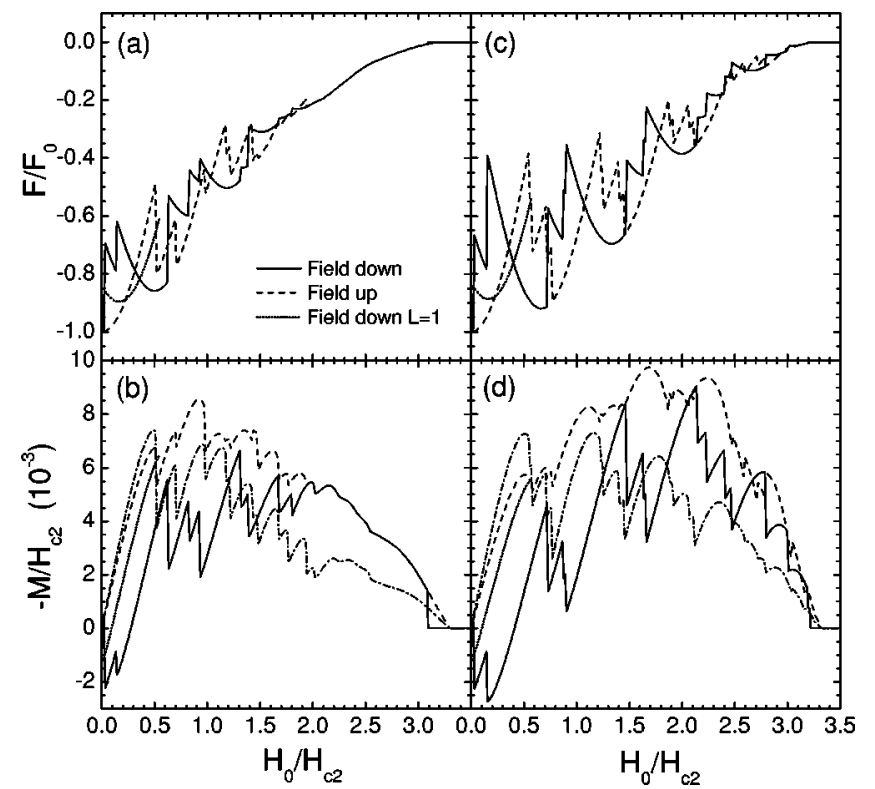

FIG. 17. The free energy and magnetization as a function of the applied magnetic field for the superconductor with three $(a, b)$ and with four $(\mathrm{c}, \mathrm{d})$ holes. Solid curves correspond to the case magnetic field sweep down and dashed curves correspond to the case magnetic field sweep up. Dotted curves indicate the $L=1$ state. The dash-dotted curves show the magnetization when the field is averaged over the area $W \times W$ for increasing field.

$=16 \rightarrow L=15, L=14 \rightarrow L=13$, and $L=10 \rightarrow L=9$ transitions. For the two top antidot sample we found only $\Delta L$ $=1$ transitions for both field sweep up and field sweep down. For the sample with two antidots along the diagonal, the magnetization calculated averaging the field over the $W$ $\times W$ area [Fig. 16(b) dash-dotted curve] is similar to that calculated only over the superconducting region [Fig. 16(b) dashed curve] and the value of the magnetization is smaller for all vortex states, except $L=0$ state. The magnetization of the sample with two antidots on the top row, calculated over the superconducting region [Fig. 16(d) dashed curve] for the $L=2$ state, is higher than for the other states, but the magnetization of this sample calculated over the $W \times W$ area [Fig. 16(d) dash-dotted curve] is maximum for the $L=0$ state. Also in this case the magnetization of the states with $L=1,2,3$ is equal.

Figure 17 shows the free energy and magnetization in increasing and decreasing fields for the superconductor with three $(a, b)$ and four $(c, d)$ holes. For the three antidot sample in increasing field we found all $\Delta L=1$ transitions except the $L=0 \rightarrow L=2$ transition in decreasing field the $L=5 \rightarrow L=3$ and $L=2 \rightarrow L=0$ transitions are also possible. For the fourantidot sample with increasing field, the vorticity changes always by 1, i.e., $\Delta L=1$. With decreasing field we find transitions where the vorticity changes from $L$ to $L-2$ for $L$ $=10,6,4,2$. The magnetization of the three-antidot sample calculated over the $W \times W$ area [Fig. 17(b) dash-dotted curve] for the Meissner state is higher than the other vortex states, while the magnetization calculated only over superconducting region [Fig. 17(b) dashed curve] is maximum for $L=3$ state.
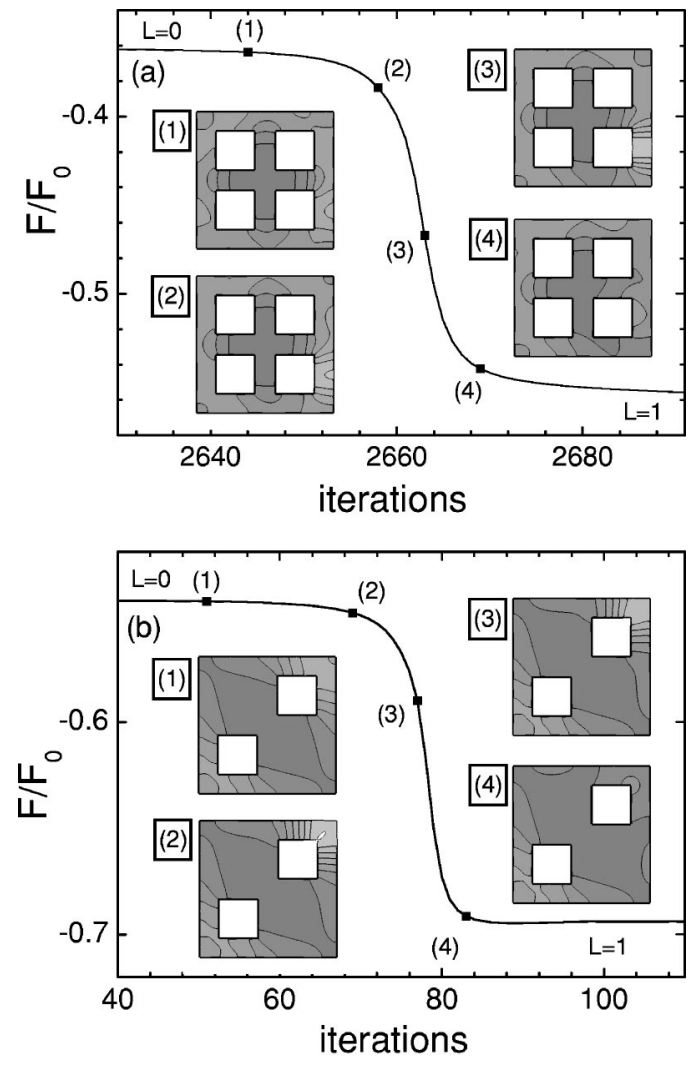

FIG. 18. The free-energy evolution during the transition from the $L=0$ state to the $L=1$ state for the superconductors with four holes (a) (at $H_{0} / H_{c 2}=0.55$ ) and with 2 holes (b) (at $H_{0} / H_{c 2}$ $=0.48$ ). In the inset, the spatial distribution of the superconducting electron density $|\psi|^{2}$ is shown after the number of iteration steps indicated by black squares.

All the considered structures have the property that they reach the $L=0$ state when the field was decreased to zero. Notice that for the samples with one, two, and three antidots the magnetization in the magnetic field region $2<H_{0} / H_{c 2}$ $<3$ is reversible which is not so for our reference sample and the one with four antidots [see Fig. 16(b)]. But notice that in all cases the $S / N$ transitions exhibits a clear hysteretic behavior. The previously discussed bunching behavior is also clearly observed, in particular for the field sweep down curves.

Next, we investigate the evolution of the free energy and the vortex state during the transition between different vortex states. This was realized as follows. For a given magnetic field our computer program calculates the free energy of the stable vortex state, corresponding to the local minimum in the free energy. When we increase the magnetic field beyond some critical value the considered vortex state no longer corresponds to a local minimum and the program runs away to a different vortex state which corresponds to a different local minimum in the free-energy space. During the iterative process, the state evolves from the initial state (which was stable up to the previous magnetic-field step) to another state, corresponding to a new local minimum of the energy for the given field.

Figures 18(a,b) show the free-energy evolution during the 


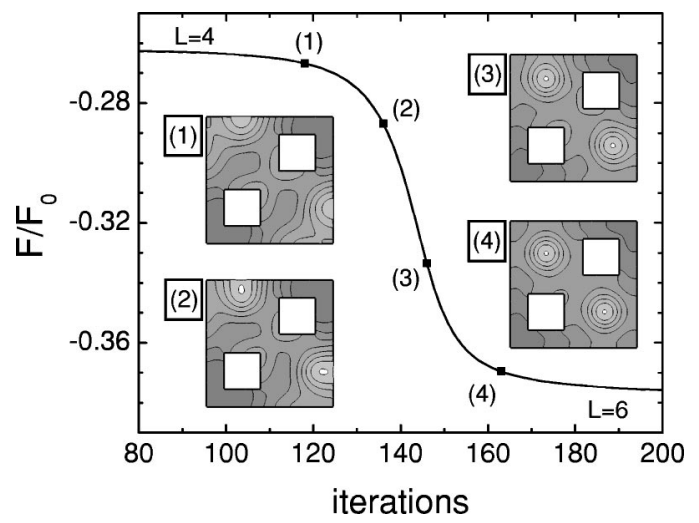

FIG. 19. The free-energy evolution during the transition between $L=4$ and $L=6$ states $\left(H_{0} / H_{c 2}=1.25\right)$ for the two-antidot sample. Cooper-pair density corresponding to the black squares in the free-energy curve is shown in the inset (dark gray regions correspond to higher density).

transition from the $L=0$ state to the $L=1$ state for the square sample with four and two holes, respectively. The insets show the evolution of the Cooper-pair density during this transition. These transitions occur at $H_{0} / H_{c 2}=0.55$ for the four-hole case and at $H_{0} / H_{c 2}=0.48$ for the two-hole case. In the case of the superconductor with four holes the first vortex enters the superconductor from the right edge into the right bottom antidot. For the superconductor with two holes the first vortex enters from the upper right corner of the superconductor. Notice that the place in the superconductor where the first vortex enters is very different in the two- and four-antidot samples. But we should stress that for the sample with two antidots such vortex entry through the corners of the sample is only found for states with vorticity not larger than 2.

The transition between the $L=4$ and the $L=6$ states for the square with two holes is also of interest. Figure 19 shows the evolution of the free energy during this transition at $H_{0} / H_{c 2}=1.25$. The contour plots of the Cooper-pair density corresponding to the black squares in the free-energy curve are shown in the inset (dark gray regions correspond to higher $|\psi|^{2}$ ). Initially there are two vortices in each antidot. Notice that vortices enter through the upper left and right bottom edges (see inset 2 of Fig. 19) at the same time and do not move towards the antidots. They become localized inside the superconductor.

An example of vortex expulsion is shown in Fig. 20 for the transition from the $L=2$ state to the $L=0$ state in the case of a superconductor containing two antidots. The insets show the spatial distribution of the superconducting electron density in the superconductor (dark regions correspond to higher density) at $H_{0} / H_{c 2}=0.05$. To transit from $L=2$ to $L=0$, the vortices in each of the antidots move at the same time in a symmetric way towards the corners of the superconductor, where they leave the sample.

\section{DEPENDENCE ON SAMPLE PARAMETERS}

Next, we investigate how our results depend on the Ginzburg-Landau parameter $\kappa$ and the sample thickness $d$.

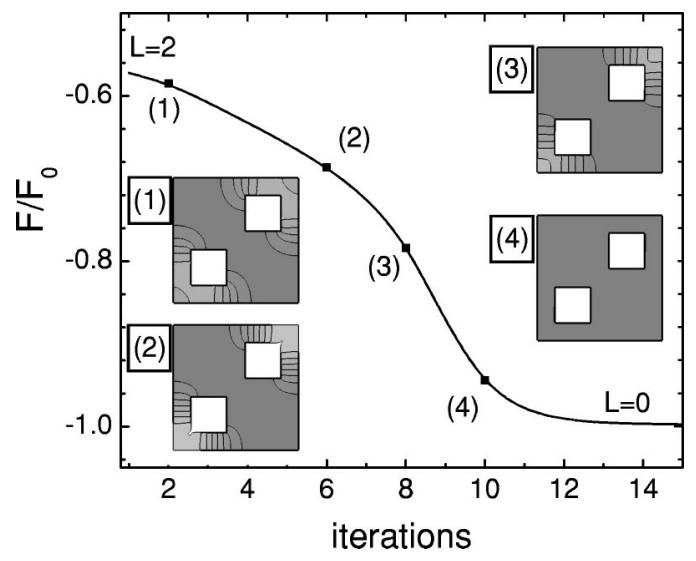

FIG. 20. The free-energy evolution during the transition from the $L=2$ to the $L=0$ state at $H_{0} / H_{c 2}=0.05$. The inset shows spatial distribution of the superconducting electron density in the superconductor (dark regions correspond to higher densities).

To limit the number of curves we will show only the results for magnetic-field sweep up.

Figure 21 shows the free energy and the magnetization for the reference sample $(a, b)$ and for the four-antidot sample for two values of the Ginzburg-Landau parameter $\kappa=0.28$ (dashed curves) and $\kappa=1.0$ (solid curves). The results for the magnetization for $\kappa=1.0$ are multiplied by 10 . The latter is the value of the Ginzburg-Landau parameter $\kappa=\lambda / \xi$ for a thin film made of $\mathrm{Pb}$. The increase of the Ginzburg-Landau parameter leads to changes in the free energy and the magnetization. The transition field between different vortex states shifts to lower fields with increasing $\kappa$. The difference is more pronounced in the magnetization where we notice that its value is more than ten times smaller in the case $\kappa=1.0$ as

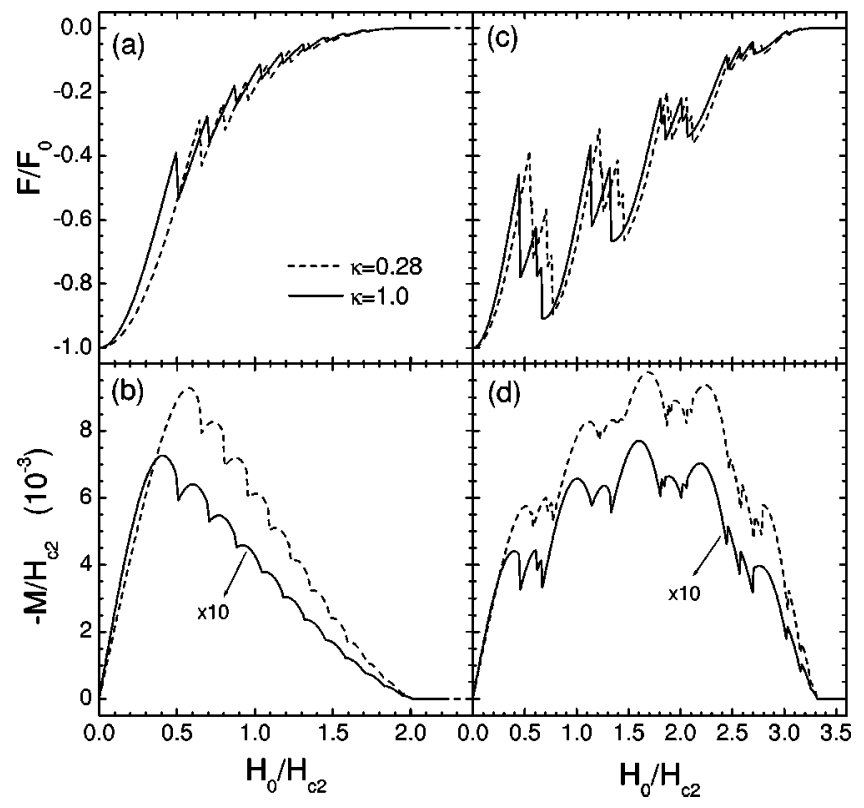

FIG. 21. The free energy and the magnetization for the reference sample $(a, b)$ and for the four-antidot sample (c,d) as a function of the magnetic field for the cases $\kappa=1.0$ (solid curves) and $\kappa=0.28$ (dashed curves). 


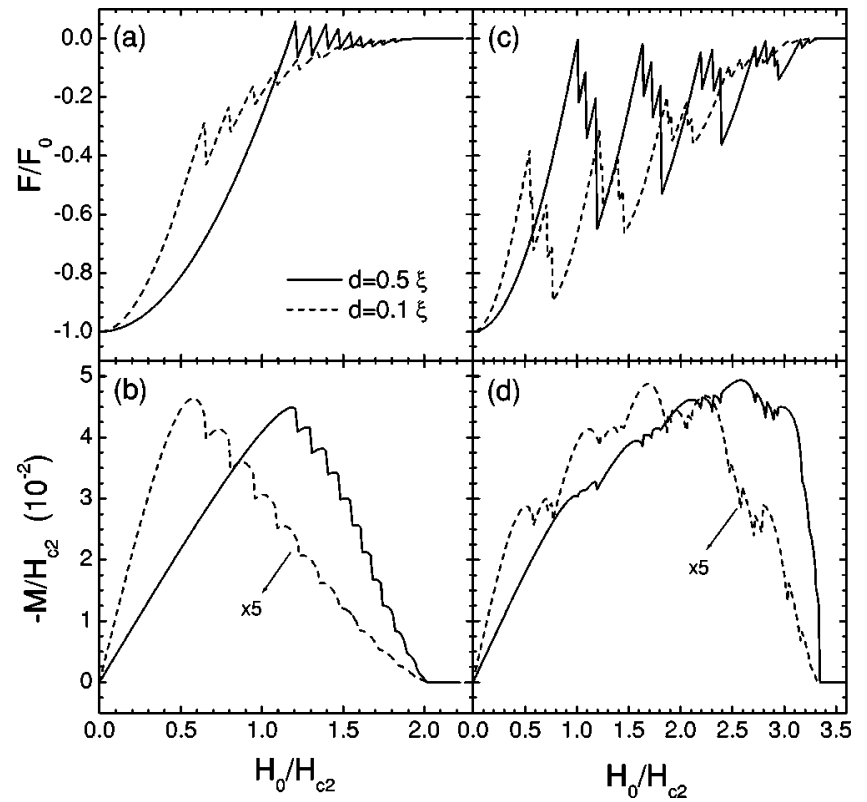

FIG. 22. The free energy and magnetization for the reference sample (a,b) and for the four-antidot sample (c,d) as a function of the magnetic field for the thickness $d=0.5 \xi$ (solid curves) and $d$ $=0.1 \xi$ (dashed curves) $(\kappa=0.28)$.

compared to the $\kappa=0.28$ case, which indicates a smaller expulsion of the magnetic field from the superconductor. A similar behavior is seen for the geometry with four holes.

The effect of the thickness of the superconductor on the vortex state is investigated in Fig. 22, where the free energy and the magnetization for the reference sample $(a, b)$ and for the four-antidot sample (c,d) are shown as a function of the applied magnetic field. The solid curves correspond to the sample thickness $d=0.5 \xi$ and the dashed curves to the thickness $d=0.1 \xi$. The results for the magnetization obtained for the thickness $d=0.1 \xi$ are multiplied by 5 . In the case of the reference sample the state with $L=0$ is more stable than the other vortex states and we can find even the Meissner state with $L=0$ with energies equal to the normal-state energy. The states with positive free energy are found also for states with $L=1,2,3,4$, and 5 . The magnetization $\left(-M / H_{c 2}\right)$ is almost five times larger for the sample with the larger thickness $d=0.5 \xi$. I the case of the four-antidot sample increasing the thickness from $d=0.1 \xi$ to $d=0.5 \xi$ leads to remarkable changes in the free energy. As we mentioned above, for the $d=0.1 \xi$ case we see all $L=n \rightarrow L=n+1$ transitions. But for the thickness $d=0.5 \xi$ there are direct transitions between the $L=2 \rightarrow L=4, L=6 \rightarrow L=8$, and $L=10 \rightarrow L=12$ states. For larger value of the sample thickness ground-state transitions between different vortex states occur at larger values of the magnetic field. Increasing $d$ stabilizes the different $L$ states up to larger magnetic fields. This is due to the increased expulsion of the applied field from the superconductor. On the other hand, in both cases the superconductor transits to the normal state at the same value of the applied magnetic field $\left(H_{0} / H_{c 2}=3.32\right)$. Such kinds of differences can also be seen from the magnetization curves. The value of the magnetization $\left(-M / H_{c 2}\right)$ is almost five times larger for the

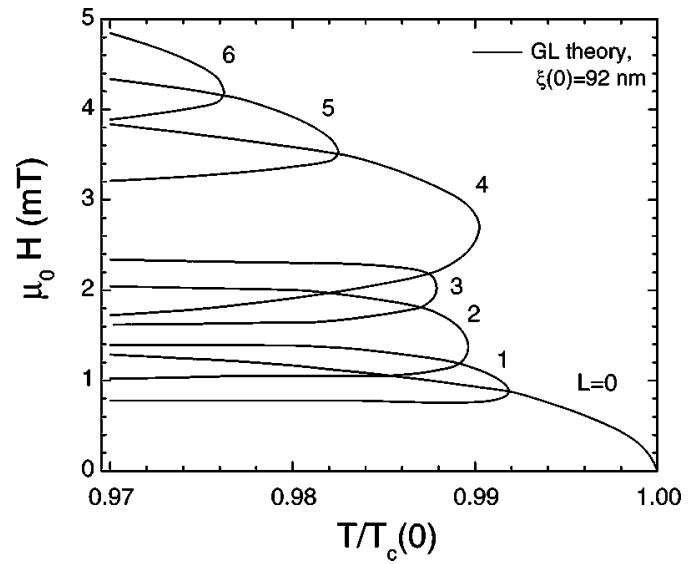

FIG. 23. The $H-T$ phase diagram and the stability area for different vortex states for the superconducting sample with four holes when $\xi(0)=92 \mathrm{~nm}$. The parameters of the superconductor are (see Fig. 1) $W=22.17 \xi(0), W_{i}=5.0 \xi(0), W_{0}=5.0 \xi(0), d=0.26 \xi(0)$, $\kappa=1.52$.

sample with increased thickness $d=0.5 \xi$. Also in this case the magnetization of the states with $L \geqslant 10$ is larger than for the other states.

\section{SUPERCONDUCTING/NORMAL PHASE DIAGRAM AND COMPARISON WITH EXPERIMENT}

In this chapter, we investigate the influence of temperature on the superconducting state in the square sample with four antidots. The temperature dependence of the coherence length $\xi$ and the magnetic field $H_{c 2}$ [see Eqs. (6)-(8)] will be included in our calculation. Therefore, the distances are now expressed in units of $\xi(0)$, magnetic field in $H_{c 2}(0)$, and temperature will be rescaled by the critical temperature $T_{c 0}$ at zero magnetic field.

In order to compare our results with the experimental ones, we used the parameters from the paper of Bruyndoncx et al. ${ }^{13}$ where they studied the nucleation of superconductivity in a unform perpendicular magnetic field in aluminum microsquares containing a few (two and four) submicron holes (antidots). They used the coherence length $\xi(0)$ $=92 \mathrm{~nm}$, and the penetration depth $\lambda(0)=140 \mathrm{~nm}$ which was found for a full square superconductor as well as for the microsquares with antidots. The parameters of our samples are as follows (see Fig. 1): $W / \xi(0)=22.17, W_{i} / \xi(0)=5.0$, $W_{0} / \xi(0)=5.0, d / \xi(0)=0.26$, and $\kappa=1.52$. The calculated $H-T$ phase boundary, using these values, is presented in Fig. 23 , which shows clear oscillations in the superconducting/ normal-state boundary. Moreover, the period of the oscillation and the peak amplitude for the state with vorticity $L$ $=4$ is larger than for the other states, which is due to a commensurability effect when the number of vortices is a multiple of the number of holes. Comparing our results with experiment (see Ref. 13), we notice a clear qualitative agreement. But the theoretical predicted $S / N$ transition at a fixed $L$ occurs at higher temperatures than observed experimentally. Also the transitions between the successive $L$ states appear at slightly larger fields in our calculations. This quantitative dis- 


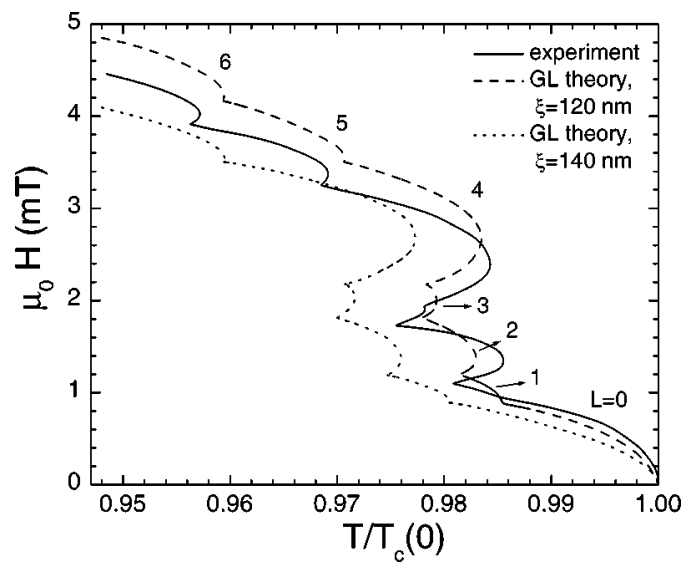

FIG. 24. The $H-T$ phase diagram for the superconducting sample with four holes for $\xi(0)=120 \mathrm{~nm}$ (dashed curve) and for $\xi(0)=140 \mathrm{~nm}$ (dotted curve). The parameters of the superconductor are (see Fig. 1) $W=17.0 \xi(0), \quad W_{i}=3.83 \xi(0), \quad W_{0}$ $=3.83 \xi(0), \quad d=0.2 \xi(0), \quad \kappa=1.17$ for $\xi=120 \mathrm{~nm}, \quad$ and $W$ $=14.57 \xi(0), \quad W_{i}=3.29 \xi(0), \quad W_{0}=3.29 \xi(0), \quad d=0.17 \xi(0), \quad \kappa$ $=1.0$ for $\xi=140 \mathrm{~nm}$. The solid curve is the experimentally obtained result.

agreement between our theory and the experiment can be due to (i) the uncertainties in the dimensions of the sample (holes), (ii) the criteria used for determining whether the sample is in the superconducting (normal) state or not, and/or (iii) the assumed value of the coherence length at zero temperature. To explore the latter possibility, we repeated the calculation and varied $\xi(0)$ keeping all other parameters fixed.

The $H-T$ phase diagram for the four-antidot superconductor is shown in Fig. 24 for the states with vorticity up to $L=6$. The solid curve was obtained experimentally in Ref. 13 and the dashed curve is the theoretically calculated $H-T$ phase diagram for $\xi(0)=120 \mathrm{~nm}$. For this value of the coherence length the correspondence is obviously much better, since the transition temperatures in our theoretical curve closely follow those from the experimental results. Still, a small difference in the transition fields exists. In our model, the transitions occur at slightly higher temperatures. This can be explained by the different criteria for the determination of the $S / N$ transition: namely, in the experiment, one assumes that superconductivity is destroyed when the region between the contacts becomes normal. In our model, for the same magnetic field, superconducting regions would still be present in the corners of the sample. Our transition fields are related to the destruction of superconductivity in the whole sample, and, therefore, they are higher than the experimental ones. In Ref. 13 the authors mentioned also another value for the coherence length $\xi(0)=140 \mathrm{~nm}$, which was deduced for the $2 \times 2$ antidot system on the basis of de GennesAlexander (dGA) model. ${ }^{7}$ The dotted curve in Fig. 24 shows the result of our calculations for such a value of $\xi(0)$. Our previous analysis still holds but now the theoretical transition temperatures are lower than the experimental ones and the transition fields are also lower.

In Fig. 25 we compare the low-magnetic-field part of the experimentally obtained $T_{c}(H)$ (solid curve) with the $T_{c}(H)$

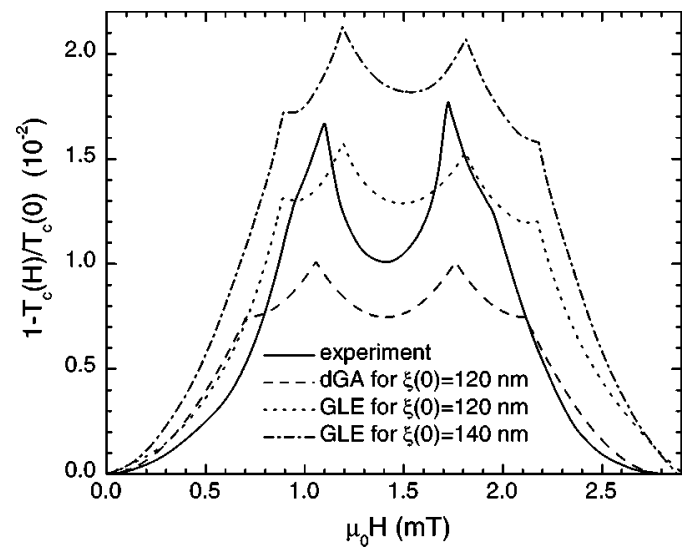

FIG. 25. Low-field part (single period) of the experimentally obtained $T_{c}(H)$ phase boundary (solid curve, Ref. 13) of the fourhole sample (where a parabolic background has been subtracted) compared with the theoretically calculated ones for $\xi(0)=120 \mathrm{~nm}$ (dotted curve) and for $\xi(0)=140 \mathrm{~nm}$ (dash-dotted curve). The dashed curve is the phase diagram calculated using the dGA model for a $2 \times 2$ cell network made of one-dimensional strips for $\xi(0)$ $=120 \mathrm{~nm}$, and the dotted curve illustrates the results of the GL simulation.

obtained in our calculations for $\xi(0)=120 \mathrm{~nm}$ (dotted curve) and for $\xi(0)=140 \mathrm{~nm}$ (dash-dotted curve), where a parabolic background

$$
1-\frac{T_{c}(H)}{T_{c}(0)}=\frac{\pi^{2}}{3}\left(\frac{\omega \xi(0) \mu_{0} H}{\Phi_{0}}\right)^{2}
$$

was subtracted. This formula was obtained in Ref. 13 for a $2 \times 2$-cell network consisting of one dimensional strips with finite width $\omega$.

We also give the $T_{c}(H)$ phase boundary calculated from the dGA model for a $2 \times 2$ cell network made of onedimensional strips (see Ref. 12) for $\xi(0)=120 \mathrm{~nm}$ (dashed curve). Although the dGA model gives a rather good qualitative agreement of the observed transition fields, our theoretical results lead to a better overall quantitative agreement. Qualitatively, the only difference is that in our calculations the transition fields between the different vortex states occur at slightly higher fields.

The vortex states with $L=1$ and $L=3$ are not clearly seen form the experimental $H-T$ diagram (only weak shoulders are seen), while they are more pronounced theoretically. To explain this we plotted in Fig. 26 the free energy of the sample at $T / T_{c 0}=0.976$, where the insets show the phase of the order parameter and the magnetic-field distribution for different $L$ states. As seen from the free-energy curve, the states with $L=2$ and $L=4$ have a large ground state region, while for the states with $L=1$ and $L=3$ this region is small. This can be explained from the contour plot of the phase of the order parameter (see insets of Fig. 26), which shows where the vortices are located. Notice that at this temperature the size of our sample is comparable to $\xi$. For the $L=1$ state the vortex is located in the center of the sample [inset (a)] and therefore the Cooper-pair density is lower in the superconducting region. For the $L=2$ state, vortices form a giant 


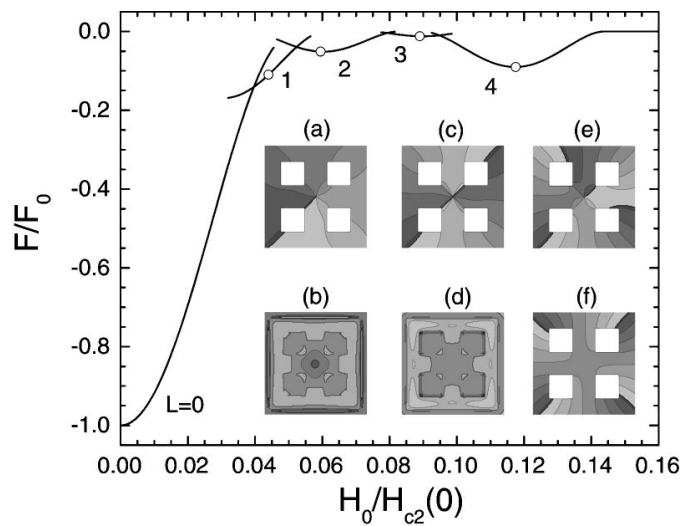

FIG. 26. The free anergy of the four-antidot sample as a function of the applied magnetic field at $T / T_{c 0}=0.976$. The insets show the phase of the order parameter (a,c,e,f) and magnetic-field distribution (b,d) for the states with $L=1$ (a,b), $L=2$ (c,d), $L=3$ (e), and $L=4$ (f). The open circles indicate the magnetic field corresponding to the insets.

vortex in the center [inset (c)], but, as the sample size is comparable to $\xi$, due to the interaction of the vortices more field goes through the holes [inset (d)], while for the $L=1$ state the magnetic field is maximum in the center of the sample [inset (b)]. Moreover, by increasing the temperature these two vortices move to the holes. For the $L=3$ state two vortices are in the holes and one is located in the center [inset (e)], which makes again the Cooper-pair density lower. For the $L=4$ state, due to the repulsion between vortices, they go through the holes [inset (f)]. Therefore, the states with $L$ $=2$ and $L=4$ are more stable than the other two states and they are more pronounced in the experimental $S / N$ phase boundary.

\section{CONCLUSIONS}

We investigated theoretically the influences of the topology of mesoscopic superconducting samples on the vortex configuration and the critical parameters. Therefore, we considered superconducting square samples containing one to four submicron antidots. We calculated the free energy of all samples as a function of the applied magnetic field, which shows the considerable influence of antidots to the number of possible vortex states, their stability, and transitions between them. For the reference sample vortex states up to $L=11$ can nucleate, while for the one-antidot sample this number is $L$ $=19$. The maximal number of vortices does not strongly depend on the number of antidots and equals $L_{\max }=19$ for all samples, except for the two-antidot sample where both antidots are located along the diagonal. In this case $L_{\max }$ $=18$. The insertion of one hole in the sample increases the $S / N$ transition field from $H_{c 3} / H_{c 2}=2.01$ to $H_{c 3} / H_{c 2}=3.21$ and the value of the $S / N$ critical field increases by $3.4 \%$ when we go from the one- to the four-antidot sample, which indicates that the size of the narrowest superconducting areas mainly determines the $S / N$ transition field. The free energy of the one-antidot sample is lower than that of the reference sample for a fixed $L$. Increasing the number of holes in the sample decreases the free energy for a fixed $L$ and groundstate transitions between different vortex states occur at lower magnetic fields. We also found that the stability of each individual superconducting state is very sensitive to the topology of the sample. For superconductors with two diagonal and four antidots, the states with even vorticity are more stable than these with odd vorticity, while this is less pronounced in the case of the two top antidot sample. For all the considered structures the vortex states show enhanced stability for commensurate vorticity, i.e., when the number of vortices is a multiple of the number of holes. However, due to the finite size of the samples this effect is less pronounced at high magnetic field. For the reference sample as well as for the four-antidot sample we found only transitions between successive $L$ states, i.e., $\Delta L=1$ in increasing field, where all transitions correspond to a jump in the free-energy curve. But for the one-, two-, and three antidot samples transitions between vortex states with high vorticity occur continuously. For the two-diagonal-antidot sample continuous transitions with $\Delta L=2$ are also possible. For decreasing magnetic field we found $\Delta L=2$ transitions for the other structures. The $S / N$ transitions exhibit a clear hysteretic behavior for the field sweep up and down. As one of the possible tools to investigate experimentally the vortex state of submicron superconducting samples, we calculated the magnetization of our samples as a function of the applied magnetic field. We found that the magnetization is strongly influenced by the presence of the antidots. For the reference sample the maximum of the magnetization correspond to the Meissner state, i.e., the $L=0$ state, while this state becomes less stable for the samples with antidots. For the one-antidot sample the largest flux expulsion is reached for $L=1$ and this is realized in the case of the two- (three-) antidot sample for $L=2(3)$, i.e., it equals the number of antidots. The sample with four antidots behaves very different and maximum magnetization is reached for $L=8$. For the sample with antidots paramagnetic response (i.e., $-M<0$ ) was found for the sates with vorticity less than or equal to the number of antidots, while for the reference sample this effect occurs for the $L=1,4,5$ states.

For the samples with antidots we also calculated the magnetization, averaging the magnetic field over the area $W$ $\times W$, which is the usual case in an experimental measurement of magnetization. More vortex states exhibit paramagnetic response and ground-state transitions to states with $-M<0$ are also possible.

More attention was given to the spatial distribution of the vortices in the superconductors, which can be interesting for practical applications. The presence of one antidot in the superconductor leads to a modification of the vortex distribution. Vortices are mainly located in the superconducting region, forming either a multivortex or a giant vortex or a combination of both vortex configurations. Superconductivity is weaker in this region than in the corner of the superconductor near the hole. This explains the continuous transitions at high fields. The interesting case is the superconductor with two antidots located along the diagonal. In this case, after the $L=5$ state, vortices are mainly located along the diagonal, where there is no antidot. At high fields 
two vortices enter the superconductor from both sides of the diagonal, which correspond to a continuous transitions with $\Delta L=2$. The samples with two antidots on the upper half of the sample and three antidots behave like the one-antidot sample. The most interesting case is the superconductor with four antidots, which has been considered to be perspective for flux quantum logic applications. In this case each second vortex is located in the opposite hole, where the first vortex was located. Notice that for all samples the maximum number of vortices that can be captured in the hole is four. We also showed the nonuniform distribution of the magnetic field inside the holes and outside the samples, and the current distribution in the superconductors.

We also investigated our samples for different values of the Ginzburg-Landau parameter and for different thicknesses. By increasing $\kappa$ the transitions between different vortex states shifts to lower fields. The influence of $\kappa$ is more pronounced in the magnetization. The magnetization decreases considerably by increasing $\kappa$, which indicates smaller expulsion of the magnetic field from the superconductor. For larger vales of the sample thickness the free energy of the vortex states are higher and transitions occur at higher fields. For the reference sample we found some vortex states with energies equal to the normal-state energy. The magnetization increases with increasing thickness of the sample.

In the last part of the paper we studied $S / N$ phase boundaries for the four-antidot sample and compared the results with experiment. The calculated $H-T$ phase diagram shows clear oscillations in the $S / N$ boundary. Contrary to the full square superconductor, in the four-antidot sample the period of the oscillations and the peak amplitude are not the same for all vortex states, which was explained by the stability of the different vortex states. We also studied the influence of the value of $\xi(0)$ on the $S / N$ boundary. The theoretically calculated $H-T$ diagram shows good agreement with the experimental results.

\section{ACKNOWLEDGMENTS}

This work was supported by the Flemish Science Foundation (FWO-Vl), the Belgian Inter-University Attraction Poles (IUAP), the "Onderzoeksraad van de Universiteit Antwerpen" (GOA), and the ESF program on "Vortex matter." We acknowledge fruitful discussions with Professor V. Moshchalkov.
*Electronic address: francois.peeters@ua.ac.be

${ }^{1}$ V.V. Moshchalkov, L. Gielen, C. Strunk, R. Jonckheere, X. Qiu, C. Van Haesendonck, and Y. Bruynseraede, Nature (London) 373, 319 (1995).

${ }^{2}$ J. Berger and J. Rubinstein, Phys. Rev. B 59, 8896 (1999).

${ }^{3}$ B.J. Baelus, F.M. Peeters, and V.A. Schweigert, Phys. Rev. B 61, 9734 (2000).

${ }^{4}$ D.Y. Vodolazov, B.J. Baelus, and F.M. Peeters, Phys. Rev. B 66, 054531 (2002).

${ }^{5}$ V.M. Fomin, V.R. Misko, J.T. Devreese, and V.V. Moshchalkov, Solid State Commun. 101, 303 (1997); Phys. Rev. B 58, 11703 (1998).

${ }^{6}$ M. Daumens, C. Meyers, and A. Buzdin, Phys. Lett. A 248, 445 (1998).

${ }^{7}$ P.G. de Gennes, C. R. Seances Acad. Sci., Ser. 2 292, 279 (1981).

${ }^{8}$ R. Rammal, T.C. Lubensky, and G. Toulouse, Phys. Rev. B 27, 2820 (1983).

${ }^{9}$ C. Ammann, P. Erdos, and S.B. Haley, Phys. Rev. B 51, 11739 (1995)

${ }^{10}$ V. Bruyndoncx, C. Strunk, V.V. Moshchalkov, C. Van Haesendonck, and Y. Bruynseraede, Europhys. Lett. 36, 449 (1996).

${ }^{11}$ T. Puig, E. Rosseel, M. Baert, M.J. Van Bael, V.V. Moshchalkov, and Y. Bruynseraede, Appl. Phys. Lett. 70, 3155 (1997).

${ }^{12}$ T. Puig, E. Rosseel, L. Van Look, M.J. Van Bael, V.V. Moshchalkov, Y. Bruynseraede, and R. Jonckheere, Phys. Rev. B 58, 5744 (1998).

${ }^{13}$ V. Bruyndoncx, J.G. Rodrigo, T. Puig, L. Van Look, V.V. Moshchalkov, and R. Jonckheere, Phys. Rev. B 60, 4285 (1999).

${ }^{14}$ V.M. Fomin, J.T. Devreese, V. Bruyndoncx, and V.V. Moshchalkov, Phys. Rev. B 62, 9186 (2000).

${ }^{15}$ B. Baelus, S.V. Yampolskii, and F.M. Peeters, Phys. Rev. B 66, 024517 (2002).
${ }^{16}$ B. Pannetier, J. Chaussy, and R. Rammal, J. Phys. (France) Lett. 44, L853 (1983); B. Pannetier, J. Chaussy, R. Rammal, and J.C. Villegier, Phys. Rev. Lett. 53, 1845 (1984).

${ }^{17}$ A. Behrooz, M.J. Burns, D. Levine, B. Whitehead, and P.M. Chaikin, Phys. Rev. B 35, 8396 (1987); F. Nori and Q. Niu, Physica B 152, 105 (1988).

${ }^{18}$ L. Van Look, B.Y. Zhu, R. Jonckheere, B.R. Zhao, Z.X. Zhao, and V.V. Moshchalkov, Phys. Rev. B 66, 214511 (2002).

${ }^{19}$ P.D. Tougaw and C.S. Lent, J. Appl. Phys. 75, 1818 (1994).

${ }^{20}$ L.N. Vu and D.J. Van Harlingen, Appl. Phys. Lett. 63, 1693 (1993).

${ }^{21}$ K. Runge and B. Pannetier, Europhys. Lett. 24, 737 (1993).

${ }^{22}$ C.C. Chi, P. Santhanam, and P.E. Blochl, J. Low Temp. Phys. 88, 163 (1992).

${ }^{23}$ S. Alexander and E. Halevi, J. Phys. (Paris) 44, 805 (1983).

${ }^{24}$ H.J. Fink, A. Lopez, and R. Maynard, Phys. Rev. B 26, 5237 (1982).

${ }^{25}$ A.K. Geim, I.V. Grigorieva, S.V. Dubonos, J.G.S. Lok, J.C. Maan, A.E. Filippov, and F.M. Peeters, Nature (London) 390, 259 (1997).

${ }^{26}$ W.A. Little and R.D. Parks, Phys. Rev. Lett. 9, 9 (1962); Phys. Rev. 133, A97 (1964).

${ }^{27}$ P.S. Deo, V.A. Schweigert, and F.M. Peeters, Phys. Rev. Lett. 79, 4653 (1997).

${ }^{28}$ V.A. Schweigert and F.M. Peeters, Phys. Rev. B 57, 13817 (1998).

${ }^{29}$ P.G. de Gennes, Superconductivity in Metals and Alloys (Addison-Wesley, New York, 1989).

${ }^{30}$ V.L. Ginzburg and L.D. Landau, Zh. Eksp. Teor. Fiz. 20, 1064 (1950).

${ }^{31}$ L.D. Landau and E.M. Lifshitz, Course of Theoretical Physics (Pergamon, Oxford, 1989), Vol. 2 and 9. 
${ }^{32}$ B.J. Baelus and F.M. Peeters, Phys. Rev. B 65, 104515 (2002).

${ }^{33}$ R. Kato, Y. Enomoto, and S. Maekawa, Phys. Rev. B 47, 8016 (1993).
${ }^{34}$ V.A. Schweigert and F.M. Peeters, Phys. Rev. B 60, 3084 (1999).

${ }^{35}$ A.K. Geim, S.V. Dubonos, I.V. Grigorieva, K.S. Novoselov, F.M. Peeters, and V.A. Schweigert, Nature (London) 407, 55 (2000). 